\title{
Magnetism and the Weiss exchange field \\ A theoretical analysis motivated by recent experiments
}

\section{Journal Article}

Author(s):

Albert, C.; Ferrari, L.; Froehlich, J.; Schlein, B.

Publication date:

2006-10

Permanent link:

https://doi.org/10.3929/ethz-b-000022835

Rights / license:

In Copyright - Non-Commercial Use Permitted

Originally published in:

Journal of Statistical Physics 125(1), https://doi.org/10.1007/s10955-006-9120-0 


\title{
Magnetism and the Weiss Exchange Field- A Theoretical Analysis Motivated by Recent Experiments
}

\author{
C. Albert, ${ }^{1,2}$ L. Ferrari, ${ }^{1,3}$ J. Fröhlich ${ }^{1}$ and B. Schlein ${ }^{1,4}$
}

Received April 28, 2005; accepted August 29, 2005

Published Online: August 5, 2006

\begin{abstract}
The huge spin precession frequency observed in recent experiments with spin-polarized beams of hot electrons shot through magnetized films is interpreted as being caused by Zeeman coupling of the electron spins to the so-called Weiss exchange field in the film. The microscopic origin of exchange interactions and of large mean exchange fields, leading to different types of magnetic order, is elucidated. A microscopic derivation of the equations of motion of the Weiss exchange field is presented. Novel proofs of the existence of phase transitions in quantum $X Y$-models and antiferromagnets, based on an analysis of the statistical distribution of the exchange field, are presented.
\end{abstract}

KEY WORDS: Magnetism, ferromagnetism, Weissfield, magnetic $\sigma$-model, meanfield theory, tight-binding models, reflection positivity, infrared bounds.

\section{INTRODUCTION}

Effects of ferromagnetism have been known since antiquity. But a mathematical understanding of the microscopic origin of ferromagnetism has remained somewhat elusive, until today! Pauli paramagnetism, ferro-, ferri- and antiferromagnetism are quantum phenomena connected to the spin of electrons and to Pauli's exclusion principle. The theory of paramagnetism in (free) electron gases is quite straightforward. ${ }^{(1)}$ Antiferromagnetism is relatively well understood: A mechanism for the generation of antiferromagnetic exchange interactions has been proposed by Anderson, ${ }^{(2)}$ who discovered a close relationship between the half-filled

\footnotetext{
${ }^{1}$ Theoretical Physics, ETH-Hönggerberg, CH-8093 Zürich

${ }^{2}$ Supported in part by the Swiss National Foundation; e-mail: albert@phys.ethz.ch

${ }^{3}$ Present address: Collegio Papio, Ascona, Switzerland

${ }^{4}$ Present address: Department of Mathematics, Stanford University, Stanford CA 94305, USA
} 
Hubbard model and the Heisenberg antiferromagnet using perturbative methods; (see also Ref. 3 for mathematically more compelling and more general variants of Anderson's key observation). It has been proven rigorously by Dyson, Lieb and Simon, ${ }^{(4)}$ using the method of infrared bounds previously discovered in Ref. 5, that the quantum Heisenberg antiferromagnet with nearest-neighbour exchange couplings exhibits a phase transition accompanied by spontaneous symmetry breaking and the emergence of gapless spin waves, as the temperature is lowered, in three or more dimensions. (The Mermin-Wagner theorem says that, in (one and) two dimensions, continuous symmetries cannot be broken spontaneously in models with short-range interactions $\left.{ }^{(6)}\right)$.

Our mathematical understanding of ferromagnetism is far less advanced. Some kind of heuristic theory of ferromagnetism emerged, long ago, in the classic works of Heisenberg, Bloch, Stoner, Dyson, Landau and Lifshitz, and others. ${ }^{(7)}$ Various insights have been gained on the basis of some form of mean-field theory, with small fluctuations around mean-field theory taken into account within a linear approximation. This approximation, however, is known to break down in the vicinity of the critical point of a ferromagnetic material, where nonlinear fluctuations play a crucial role. ${ }^{(8)}$

In a variety of tight-binding models of itinerant electrons, ferromagnetic order has been exhibited in the ground state (i.e., at zero temperature); see Refs. 9-11. One of these models is a fairly natural two-band model in which ferromagnetism arises from a competition between electron hopping, Coulomb repulsion and an on-site Hund's rule. ${ }^{(11)}$ (Hund's rule says that the spin-triplet state of two electrons occupying the same site is energetically favoured over the spin-singlet state. It should be emphasized, however, that a mathematically rigorous derivation of Hund's rule in atomic physics from first principles has not been accomplished, so far.) None of the results in Refs. 9-11 comes close to providing some understanding of ferromagnetic order and of an order-disorder phase transition at positive temperature. It is not known how to derive, with mathematical precision, an effective Hamiltonian with explicit ferromagnetic exchange couplings from the microscopic Schrödinger equation, or a tight-binding approximation thereof, of ferromagnetic materials. But even if we resort to a phenomenological description of such materials in terms of models where ferromagnetic exchange couplings have been put in by hand we face the problem that we are unable to exhibit ferromagnetic order at low enough temperature and to establish an order-disorder phase transition in three or more dimensions. No mathematically rigorous proof of the phase transition in the quantum Heisenberg ferromagnet is known, to date! (Such a result has, however, been established for classical Heisenberg models in Ref. 5).

$\mathrm{Ab}$-initio quantum Monte Carlo simulations of models of quantum ferromagnets are plagued by the well known "sign- (or complex-phase) problem." 
Thus, until now, there are neither substantial mathematically rigorous results on, nor are there reliable ab-initio numerical simulations of, realistic models of ferromagnetic metals, such as $\mathrm{Ni}$, Co or Fe! Given that ferromagnetism is among the most striking macroscopic manifestations-apparent, e.g., in the needle of a compass-of the quantum-mechanical nature of matter, this is clearly a desolate state of affairs.

In the present paper we shall not remedy this unsatisfactory situation. However, first, we attempt to draw renewed attention to it, and, second, we outline a formalism and some fairly elementary analytical observations of which we hope that they will ultimately lead to a better, mathematically rather precise understanding of ferromagnetism. Were it not known already, our analysis and the one in Ref. 11 would make clear that ferromagnetism is a non-perturbative phenomenon involving strong correlations and gapless modes. To understand it mathematically will most probably necessitate a full-fledged multi-scale (renormalization group) analysis. The formalism presented in this paper and our calculations are intended to provide a convenient starting point for such an analysis.

Analytical work on ferromagnetism may seem to be rather unfashionable. However, there are recent developments, such as spintronics, fast magnetic devices, etc. that may make work like ours appear worthwhile. Our own motivation for the work that led to this paper actually originated in studying recent experiments with beams of spin-polarized, hot electrons shot through ferromagnetically ordered films consisting of $\mathrm{Ni}$, $\mathrm{Co}$ or $\mathrm{Fe}$ that were carried out in the group of H.C. Siegmann at ETH; see Refs. 12, 13. Back in 1998, it became clear to one of us that the concept of the "Weiss exchange field" (see e.g. Refs. 14, 15) would play a useful role in a theoretical interpretation of the experimental results reported in Refs. 12, 13. More generally, the Weiss exchange field appears to offer a key to a systematic study of phase transitions in magnetic materials, magnetic order, spin precession, magnon dynamics and electron transport in magnetic materials; (see Sec. 2 for a brief overview). In this paper, we focus attention on elucidating the microscopic origin of the Weiss exchange field, on the role it plays in the theory of magnetism, and on its dynamics.

Our paper is organized as follows.

In Sec. 2, we describe the experiments reported in Refs. 12, 13 and sketch a phenomenological interpretation, based on scattering theory, of the results found in these experiments, merely adding some conceptual remarks to the discussion of our experimental colleagues and describing the role played by the Weiss exchange field.

A mathematically precise analysis of the scattering of electrons (or neutrons, photons, ... ) at dynamical targets, such as magnetic films, metallic solids, liquid droplets, ..., will appear elsewhere; (some first results appear in Ref. 16).

We then briefly mention some further experiments involving spin-polarized electric currents in magnetic materials that can be interpreted, theoretically, by 
introducing a Weiss exchange field. Furthermore, some "gedanken experiments," such as a Stern-Gerlach experiment for electrons, providing direct evidence for effects caused by the exchange field are outlined.

In Sec. 3, we reformulate one-band $t-J$ and Hubbard models in terms of a dynamical Weiss exchange field. For this purpose, the standard imaginary-time functional integral formalism for the analysis of thermal equilibrium states of quantum many-body systems is recalled. The Weiss exchange field is seen to be a Lagrange-multiplier field in a Hubbard-Stratonovich transformation of the original functional integral that renders the action functional quadratic in the Grassmann variables describing the electronic degrees of freedom; see e.g. Refs. 15, 17. The effective field theory of the Weiss exchange field is obtained after integrating over those Grassmann variables. The effective (imaginary-time) action functional of the exchange field and identities for Green functions of spin operators are derived.

In Sec. 4, we determine the leading terms of the effective action of the Weiss exchange field, $\mathbf{W}$, in the approximation where fluctuations of the length of the exchange field are neglected. For this purpose, we derive the ferro- and antiferromagnetic mean-field equations from the exact effective action of the exchange field. By solving these equations we determine the most likely length, $W_{0}$, of the exchange field. From that point on, the length of the exchange field is frozen to be $|\mathbf{W}| \equiv W_{0}$.

We then consider a one-band Hubbard model with a half-filled band and find that, in this situation, the effective action of $\mathbf{W}$ is the one of a nonlinear $\sigma$ model with a minimum that favours Néel order. This result is found on the basis of controlled perturbative calculations and goes beyond linear stability analysis of the antiferromagnetic mean-field solution, (which has been presented, e.g., in Ref. 15). It represents a functional-integral version of Anderson's basic observations. ${ }^{(2)}$

We then turn to ferromagnetically ordered mean field solutions and show that translation-invariant, but time-dependent fluctuations are not a source of instability of such a solution. Then we consider a one-band Hubbard model with a less than half-filled, fairly flat band. One expects that ferromagnetism prevails under these conditions. Indeed, we find that, at low temperatures, the ferromagnetic mean-field equation has a non-trivial solution, and that this solution belongs to a quadratically stable critical point of the effective action of $\mathbf{W}$. This conclusion is the result of somewhat subtle calculations involving processes close to the Fermi surface, which make the dominant contribution (but would lead to small-energy denominators in a purely perturbative analysis). Details will appear in Ref. 18. Our calculations support the idea that the one-band Hubbard model with a less than half-filled, fairly flat band describes coexistence of metallic behaviour with ferromagnetic order, at sufficiently low temperatures. A similar conclusion was reached, tentatively, in Ref. 11 for some two-band Hund-Hubbard models. The methods of the present paper also apply to the model discussed in Ref. 11; see Ref. 18. 
In the next to last subsection of Sec. 4, we exhibit a universal Wess-Zumino term in the effective action of the Weiss exchange field $\mathbf{W}$ and calculate its coefficient, which is purely imaginary. The Wess-Zumino term is "irrelevant" for antiferromagnets, but plays a crucial role in the dynamics of magnons in ferromagnetically ordered systems. Repeating arguments in Ref. 19, we derive the Landau-Lifshitz equations for magnons in a ferromagnet.

Finally, we draw attention to two well known arguments explaining why there is no magnetic ordering at positive temperature, in one and two dimensions; (but see Ref. 6).

In Sec. 5, we sketch novel rigorous proofs, based on analyzing the effective field theory of the exchange field, $\mathbf{W}$, of the existence of phase transitions and magnetic order at low temperatures in a class of $X Y$-models, Heisenberg antiferromagnets and ferromagnets of localized $S O(2 n)$-spins, for $n=1,2, \ldots$ See Ref. 4 for the original results. Our proof is based on establishing reflection positivity of the effective field theory of the exchange field $\mathbf{W}$ and then using the original techniques developed in Ref. 5; (see also Refs. 20, 21).

It should be emphasized that the concept of the Weiss exchange field has a number of further, quite exciting applications. We hope to return to these matters in future papers.

Some of the material in this paper has a review character; but some of it is new. We hope it is fairly easy to read. If it draws renewed attention to some of the deep technical problems in the quantum theory of magnetism it has fulfilled its purpose. We gratefully dedicate this paper, belatedly, to two great colleagues and friends of the senior author (J.F.): G. Jona-Lasinio, on the occasion of his seventieth birthday, and H.-C. Siegmann, on the occasion of his retirement from ETH.

\section{REAL AND GEDANKEN EXPERIMENTS INVOLVING THE WEISS EXCHANGE FIELD}

We start this section with a brief description of recent experiments carried out by Oberli, Burgermeister, Riesen, Weber and Siegmann at ETH-Zürich. ${ }^{(12,13)}$ In these experiments, a beam of hot, spin-polarized electrons is shot through a thin ferromagnetic film ( $\mathrm{Ni}, \mathrm{Co}$, or $\mathrm{Fe}$ ) and the polarization of the outgoing beam is observed. Their experimental setup is as described in Fig. 1.

The following quantities are measurable:

(i) The thickness, $d$, of the film; $d$ is a few nanometers.

(ii) The average energy, $E$, of an incident electron; if $E_{F}$ denotes the Fermi energy of the magnetic film then $E-E_{F}$ varies between $4 \mathrm{eV}$ and $16 \mathrm{eV}$. The group velocity of the electrons inside the film is denoted by $\mathbf{v}$; it is not directly measurable, but it is comparable to $\left(2(E+e V) / m_{\mathrm{el}}^{*}\right)^{1 / 2}$, 

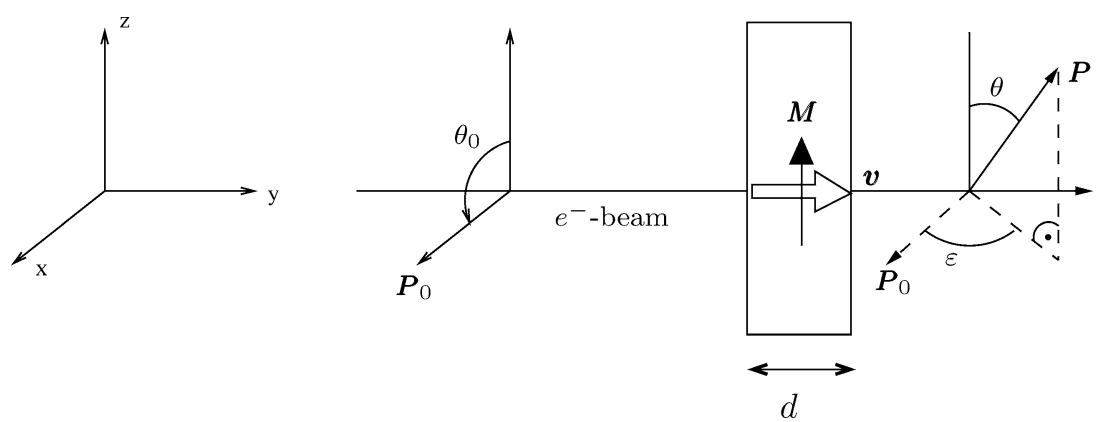

Fig. 1. Experimental setup.

where $e V$ is the average potential energy of an electron and $m_{\mathrm{el}}^{*}$ its effective mass inside the film.

(iii) The degree, $P_{0}$, and the direction, $\mathbf{n}_{0}$, of the spin polarization of the incident electron beam; (in Fig. 1, $\mathbf{n}_{0}$ is parallel to the $x$-direction, $\mathbf{v}$ to the $y$-direction); the same quantities, $P$ and $\mathbf{n}$, for the outgoing beam.

(iv) The direction of the magnetization, $\mathbf{M}$, of the film (in Fig. 1 chosen to be parallel to the $z$-axis); the angles, $\theta_{0}$ and $\theta$, between $\mathbf{n}_{0}$ and $\mathbf{M}$ and between $\mathbf{n}$ and $\mathbf{M}$, respectively $\left(\theta_{0}=\pi / 2\right.$, in Fig. 1). Experimentally, the angle $\theta$ is found to be considerably smaller than $\theta_{0}$, i.e., the spins of the transmitted electrons rotate into the direction of the spontaneous magnetization $\mathbf{M}$ of the film. This is interpreted as being mainly due to an enhanced absorption of minority-spin electrons, as compared to majority-spin electrons; see (vi). (It appears that the contribution of spin flip processes accompanied by magnon emission into the film - "Stoner excitations" - to the total spin rotation is only around $5 \%{ }^{(12)}$ ).

(v) The spin precession angle, $\epsilon$, between the projections of $\mathbf{n}_{0}$ and of $\mathbf{n}$ onto the plane perpendicular to $\mathbf{M}$ (the $x y$-plane of Fig. 1 ); $\epsilon$ is found to be "large" (tens of degrees).

(vi) Let $I$ be the intensity of the incident beam, and let $I^{+}=I^{+}(E)$ and $I^{-}=I^{-}(E)$ be the intensities of the outgoing beam of electrons with spin parallel or antiparallel to $\mathbf{M}$, respectively, assuming the incident beam has intensity $I$ and the spins of its electrons are parallel to $\mathbf{M}$ $\left(\mathbf{n}_{0}\right.$ parallel to $\left.\mathbf{M}, P_{0} \cong 1\right)$, or antiparallel to $\mathbf{M}\left(\mathbf{n}_{0}\right.$ anti-parallel to $\mathbf{M}$, $P_{0} \cong 1$ ), respectively. Then $\theta_{0}=\theta=0$, or $\theta_{0}=\theta=\pi$, respectively, and $\epsilon=0 . I^{+}$and $I^{-}$can be measured and yield the spin-transmission asymmetry

$$
A=\frac{I^{+}-I^{-}}{I^{+}+I^{-}}
$$


$A$ is found to be positive and large. This is interpreted in terms of rates of transitions of electrons into unoccupied $3 \mathrm{~d}$ states (holes): There are more unoccupied $3 \mathrm{~d}$ states in the film with spin antiparallel to $\mathbf{M}$ (minority spin) than with spin parallel to $\mathbf{M}$ (majority spin). This explains qualitatively the experimental results found for $A$ and for $\theta-\theta_{0}$ (see (iv) $)^{(12)}$.

(vii) The orbital deflection angle, $\alpha$, between the directions of the incident and the transmitted beam (not indicated in Fig. 1). Experimentally $\alpha$ is found to be negligibly small. This tells us that the integrated Lorentz force on the electrons transmitted through the film is tiny. The precession of the spins of the electrons when they traverse the film can therefore not be explained by Zeeman coupling of the spins to the magnetic field inside the layer. It is mainly due to Zeeman coupling of the spins to what will be called the Weiss exchange field. In iron, the Weiss exchange field causing the observed spin precession would correspond to a magnetic field of roughly 8000 Tesla (which is gigantic).

A theoretical interpretation of the experimental results reported in Ref. 12 can be attempted within the formalism of scattering theory. If the luminosity of the incident beam is low we can consider a single incoming electron. The incoming state is described as a tensor product of a Pauli spinor, $\psi_{\text {in }}$, describing the incident electron and a state, $\xi$, of the film. Typically, $\xi$ is the ground state (temperature $T=0)$ or a thermal equilibrium state $(T>0)$ of the film. The outcoming state, long after the interactions between an outgoing electron and the film have taken place, is more complicated and will, in general, exhibit entanglement between the electron and the degrees of freedom of the film. If only measurements far away from the film are performed, as in Ref. 12, the outgoing state can be described as a density matrix

$$
P_{\text {out }}=\left(\rho_{N}\right)_{N=0}^{\infty},
$$

where $\rho_{N}$ is a non-negative, trace-class operator on the Hilbert space of $N$ outgoing electrons (the incident electron has knocked $N-1$ electrons out of the film), $N=2,3, \ldots ; \rho_{0}$ is a non-negative number, the absorption probability, $\rho_{1}$ is a non-negative trace-class operator on the Hilbert space

$$
\mathcal{H}=L^{2}\left(\mathbb{R}^{3}\right) \otimes \mathbb{C}^{2}
$$

of square-integrable Pauli spinors and describes the (generally mixed) state of one outgoing electron. "Conservation of probability" implies that

$$
\rho_{0}+\sum_{N=1}^{\infty} \operatorname{Tr} \rho_{N}=1 .
$$


The state $P_{\text {out }}$ is obtained by taking a partial trace of the outgoing state of the total system, including the film, over the degrees of freedom of the film. This is justified, because the degrees of freedom of the film are not observed in the experiment. If the energy, $E$, of the incident electron is below (or comparable to) the threshold, $\Sigma^{(2)}$, for emission of two or more electrons from the film then

$$
\rho_{N}=0 \quad \text { for } \quad N \geq 2 .
$$

In the interpretation of the experimental data provided in Ref. 12 , this is tacitly assumed.

Experimentally, the absorption probability $\rho_{0}$ and the spin polarizations $\mathbf{P}_{0}$ and $\mathbf{P}$ of the incoming and the outgoing electron, respectively, are measured. The vectors $\mathbf{P}_{0}$ and $\mathbf{P}$ are given by

$$
\mathbf{P}_{0} \equiv P_{0} \mathbf{n}_{0}=\left\langle\psi_{\text {in }}, \boldsymbol{\sigma} \psi_{\text {in }}\right\rangle,
$$

and

$$
\mathbf{P} \equiv P \mathbf{n}=\frac{1}{\operatorname{Tr}_{\mathcal{H}}\left(\rho_{1}\right)} \operatorname{Tr}_{\mathcal{H}}\left(\rho_{1} \boldsymbol{\sigma}\right),
$$

where $\psi_{\text {in }}$ is the wave function of the incident electron, $\sigma=\left(\sigma_{x}, \sigma_{y}, \sigma_{z}\right)$ is the vector of Pauli matrices, and $\widetilde{\rho}_{1}:=\left[\operatorname{Tr}_{\mathcal{H}}\left(\rho_{1}\right)\right]^{-1} \rho_{1}$ is the conditional state of the outgoing electron, given that it has not been absorbed in the film. If (2.4) is assumed to hold then

$$
\operatorname{Tr}_{\mathcal{H}}\left(\rho_{1}\right)=1-\rho_{0} .
$$

Since we have assumed that the incoming electron has been prepared in a pure state,

$$
P_{0} \equiv\left|\mathbf{P}_{0}\right|=1 .
$$

However, there is, a priori, no reason why $\widetilde{\rho}_{1}$ should be a pure state. If it were pure then

$$
P=|\mathbf{P}|=1 .
$$

It would be highly interesting to estimate, experimentally, the amount of entanglement with the film (or decoherence) in the state of the outgoing electron by measuring the quotient $P / P_{0}$. If $P / P_{0}<1$ then $\widetilde{\rho}_{1}=\left[\operatorname{Tr}_{\mathcal{H}}\left(\rho_{1}\right)\right]^{-1} \rho_{1}$ is $n o t$ a pure state, anymore, meaning there is entanglement with the film. Apparently, $P / P_{0}$ has not been measured accurately, yet.

A moment's reflection shows that spin flip processes accompanied by magnon emission in the film ("Stoner excitations") lead to entanglement; while absorption of electrons into unoccupied $3 \mathrm{~d}$ states need not be correlated with entanglement of the states of those electrons that $d o$ traverse the film. In fact, it is implicitly assumed in Ref. 12 that if Stoner excitations are neglected then $\widetilde{\rho}_{1}$ is close to a 
pure state (at least in spin-space). The experimental techniques of Ref. 12 could be used to test this hypothesis.

Next, we express the spin transmission asymmetry $A$ (see (vi)) in terms of outgoing states. Let $\psi_{\text {in }}^{+}$and $\psi_{\text {in }}^{-}$be incoming states with spin polarization $\mathbf{P}_{0} \equiv \mathbf{P}_{0}^{+}$ parallel to $\mathbf{M}$ (majority spin) and $\mathbf{P}_{0} \equiv \mathbf{P}_{0}^{-}$antiparallel to $\mathbf{M}$ (minority spin). Let $P_{\text {out }}^{+}=\left(\rho_{N}^{+}\right)_{N=0}^{\infty}$ and $P_{\text {out }}^{-}=\left(\rho_{N}^{-}\right)_{N=0}^{\infty}$ be outgoing states corresponding to $\psi_{\text {in }}^{+}, \psi_{\text {in }}^{-}$, respectively. Then

$$
A=\frac{\rho_{0}^{-}-\rho_{0}^{+}}{2-\rho_{0}^{+}-\rho_{0}^{-}},
$$

as follows from (2.3). More interesting would be measurements of

$$
\mathbf{P}^{ \pm}=\operatorname{Tr}\left(\widetilde{\rho}_{1}^{ \pm} \boldsymbol{\sigma}\right)
$$

Clearly $\mathbf{P}^{ \pm}$are parallel or anti-parallel to $\mathbf{M}$; but their lengths $P^{ \pm}=\left|\mathbf{P}^{ \pm}\right|$ought to be measured. In Ref. 12 , it is tacitly assumed that $P^{ \pm} \cong 1$, and that the states $\widetilde{\rho}_{1}^{ \pm}$ are close to pure states; but serious experimental data backing up this hypothesis appear to be lacking. It is clear that it would be invalidated if "Stoner excitations" played an important role.

In the following, we outline a phenomenological description of the experiments in Ref. 12, assuming that (2.4) and the hypothesis just discussed (purity of $\tilde{\rho}_{1}^{ \pm}$) are valid. (A more detailed discussion of the scattering approach to electron transmission- and reflection experiments will be presented elsewhere.)

When an incoming electron enters the film it occupies an empty state of the film. If the film is crystalline this state belongs to a band of states; let $\alpha$ be the corresponding band index. The state of an electron in band $\alpha$ is described by a Pauli spinor

$$
\phi=\left(\phi^{+}, \phi^{-}\right),
$$

where $\phi^{+}$and $\phi^{-}$are the components of $\phi$ with spin parallel or anti-parallel to the magnetization $\mathbf{M}$, respectively. Adopting the approximation of the Peierls substitution, the Pauli equation for $\phi$ in configuration space has the form

$$
i \hbar \mathcal{D}_{0} \phi=\mathcal{E}_{\alpha}(-i \hbar \mathcal{D}) \phi,
$$

where $\mathcal{E}_{\alpha}(\mathbf{p})$ is the band function of band $\alpha$,

$$
\begin{aligned}
& \mathcal{D}_{0}=\frac{\partial}{\partial t}+i \frac{e}{\hbar} \phi^{c}+i\left(\mathbf{W}_{0}^{c} \cdot \frac{\sigma}{2}\right), \\
& \mathcal{D}_{j}=\frac{\partial}{\partial x^{j}}+i \frac{e}{\hbar} A_{j}+i\left(\mathbf{W}_{j} \cdot \frac{\boldsymbol{\sigma}}{2}\right) .
\end{aligned}
$$

Here $\phi^{c}$ denotes a (complex) electrostatic potential, $\mathbf{W}_{0}^{c}$ is a (complex) Weiss exchange field, $\left(A_{j}\right)=\left(A_{1}, A_{2}, A_{3}\right)$ is the electromagnetic vector potential, and 
$\left(\mathbf{W}_{j}\right)=\left(\mathbf{W}_{1}, \mathbf{W}_{2}, \mathbf{W}_{3}\right)$ is an $S U(2)$-vector potential describing spin-orbit interactions. As discussed in Ref. 14, (2.13) displays electromagnetic U(1)-gauge invariance and $S U(2)$-gauge invariance, i.e., covariance with respect to local $S U(2)$-rotations in spin space. A number of important consequences of these gauge symmetries have been pointed out in Ref. 14 .

According to (vii) above, effects of the electromagnetic vector potential A are apparently negligible; so $\mathbf{A}$ is set to 0 . The electrostatic vector potential $\phi^{c}$ is given, approximately, by

$$
\phi^{c}=V+i \hat{v}
$$

where $e V$ is the surface exit work, and the imaginary part, $\hat{v}$, of $\phi^{c}$ provides a phenomenological description of spin-independent inelastic absorption processes inside the film. Velocity-dependent spin-flip processes due to spin-orbit interactions appear to play a very minor role in the experiments reported in Ref. 12; so we may set $\mathbf{W}_{j}$ to $0, j=1,2,3$.

The Weiss exchange field $\mathbf{W}_{0}^{c}$ is given by

$$
\mathbf{W}_{0}^{c}=\mathbf{W}-i \mathbf{w}
$$

where the real part $\mathbf{W}$ describes exchange interactions between the incoming electron and the electron density of the film, and the imaginary part, $\mathbf{w}$, yields a phenomenological description of spin-dependent absorption processes.

Let $\phi_{\text {in }}$ denote the Pauli spinor describing the state of an electron when it enters the film at some time $t_{0}$. Equation (2.13) can be solved for $\phi=\phi_{t}, t \geq t_{0}$, with $\phi_{t_{0}}=\phi_{\text {in. }}$. The solution is explicit if $\mathbf{A}=0, \mathbf{W}_{j}=0, j=1,2,3$. Let us suppose that the real part $\mathbf{W}$ and the imaginary part $\mathbf{w}$ of the exchange field $\mathbf{W}_{0}^{c}$ are both anti-parallel to the magnetization $\mathbf{M}$ of the film. Then (2.13) leads us to consider two simple, quasi-one-dimensional scattering problems in a complex potential well of depth

$$
e V+i e \hat{v} \pm \frac{1}{2}(\Omega-i \omega)
$$

with $\Omega=|\mathbf{W}|$ and $\omega=|\mathbf{w}|$, for electrons with spin parallel to $\mathbf{M}(+)$, or antiparallel to $\mathbf{M}(-)$, respectively. The solution to these scattering problems can be found in every book on elementary quantum mechanics. For the purposes of interpreting the results in Ref. 12, a semi-classical treatment appears to be adequate. The group velocity, $\mathbf{v}_{ \pm}$, of an incoming electron wave with energy peaked at $E$ and spin up (+), or down (-), inside the film can be found by solving the equation

$$
\mathcal{E}_{\alpha}(\mathbf{p})=E-e V \mp \frac{1}{2} \Omega
$$


for $\mathbf{p}$ and then setting

$$
\mathbf{v}_{ \pm}=\frac{\partial \mathcal{E}_{\alpha}}{\partial \mathbf{p}}\left(\mathbf{p}^{ \pm}\right)
$$

where $\mathbf{p}^{ \pm}$is a solution of (2.19) chosen such that $\mathbf{v}_{ \pm}$points in the positive $y$-direction. The sojourn time $\tau_{ \pm}$of the wave inside the film is then given by

$$
\tau_{ \pm}=\frac{d}{v_{ \pm}}, \quad \text { with } \quad v_{ \pm}=\left|\mathbf{v}_{ \pm}\right|
$$

If $\phi_{\text {in }}^{ \pm}$is the state of the electron with spin up $(+)$, or spin down $(-)$, respectively, when it enters the film its state $\phi_{\text {out }}^{ \pm}$upon leaving the film is then given, approximately, by ${ }^{5}$

$$
\phi_{\text {out }}^{ \pm} \cong \exp \left(-i \tau_{ \pm}\left[(E-e V)-i e \hat{v} \mp \frac{1}{2}(\Omega-i \omega)\right]\right) \phi_{\text {in }}^{ \pm} .
$$

The presence of inelastic absorption processes implies that $e \hat{v} \mp \omega / 2>0$. Setting

$$
\exp \left(-\tau_{ \pm}[\hat{v} \mp \omega / 2]\right)=c \sqrt{1 \pm A}
$$

we find that

$$
\phi_{\text {out }}^{ \pm}=c \sqrt{1 \pm A} e^{i(\theta \pm \epsilon / 2)} \phi_{\text {in }}^{ \pm},
$$

where $A$ is the spin transmission asymmetry (see point (iv) and (2.10)), $\epsilon$ is the spin precession angle, and $\theta$ is an (unimportant) spin-independent phase. If $v_{+} \cong v_{-}=v$ then $\tau_{+} \cong \tau_{-} \cong d / v$, and (2.22) yields

$$
\epsilon \cong \tau_{ \pm} \Omega \cong(d / v)|\mathbf{W}|
$$

Thus, measuring $\epsilon$ and $d$ and estimating $v$ yields an approximate value for the size of the spin precession angular velocity $\Omega$ and hence of the size of the Weiss exchange field.

Equations (2.22) and (2.24) could be mistaken for equations describing kaonor neutrino oscillations and are analogous to the equations describing the Faraday rotation of light traversing a magnetized medium.

The origin of the Weiss exchange field $\mathbf{W}$ is hardly a mystery: The spin of an electron traversing the film in a band $\alpha$ apparently experiences (exchange) interactions with the spins of the occupied states of the film. Since states with spin up and with spin down are occupied asymmetrically (corresponding to the fact that $\mathbf{M} \neq 0$ ), the net spin density, $\mathbf{S}(x)$, at a point $x$ in the film is different from zero. The Weiss exchange field $\mathbf{W}=\mathbf{W}(x)$ is given by

$$
\mathbf{W}(x)=-J_{\alpha} \mathbf{S}(x) \text {, }
$$

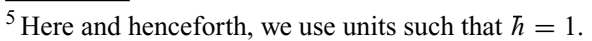


where $J_{\alpha}$ is the strength of the exchange coupling between spins in the $\alpha^{\text {th }}$ band and those in the occupied band. The theoretical discussion above is based on a mean field ansatz: The exchange field $\mathbf{W}$ in (2.14), (2.17) is chosen to be

$$
\mathbf{W}=\langle\mathbf{W}(x)\rangle=-J_{\alpha}\langle\mathbf{S}(x)\rangle .
$$

(Our conventions are such that $\langle\mathbf{S}(x)\rangle$ is parallel to the magnetization $\mathbf{M}$.) One of the surprising implications of the experimental results of Ref. 12 is that, apparently, $J_{\alpha}$ is quite large, even for rather high-lying bands $(\alpha)$, implying that the orbitals of states in such bands must have substantial overlap with those of states in the partially occupied, spin-polarized band.

Equation (2.26) makes it clear that $\mathbf{W}(x)$ is a dynamical field, its dynamics being described by the Landau-Lifshitz equation. Variations of $\mathbf{W}$ in space and time describe spin waves, its field quanta are the magnons.

One of the main purposes of this paper is to derive the effective quantum dynamics of $\mathbf{W}(x)$ within a Lagrangian functional integral formalism and to sketch what can be accomplished with this formalism; see Secs. 3 and 4.

We conclude this section by drawing the readers' attention to various further effects involving spin-polarized electric currents or electron beams in magnetic materials that can be interpreted neatly with the help of the Weiss exchange field; (a more detailed discussion goes beyond the scope of this paper).

Among such effects is the phenomenon of giant magneto resistance, see Ref. 22; ones due to interactions of a spin-polarized current with spin waves, such as Gilbert damping ${ }^{(23)}$ and negative Gilbert damping ${ }^{(24)}$; local switching of the direction of $\mathbf{W}$ caused by its interactions with the precessing spins of electrons in a spin-polarized beam, ${ }^{(25)}$ polarization-dependent transmission of a spin-polarized electron beam through a magnetized sample, which can be used for the purpose of imaging magnetic domain structures (BEMM), see Ref. 26; etc.

We finally sketch some "gedanken experiments."

By applying an external magnetic field rotating in the $x y$-plane with angular velocity $\omega_{0}$ to a film magnetized in the $z$-direction, the exchange field $\mathbf{W}$ can be made to rotate around the $z$-axis:

$$
\mathbf{W}(t)=W\left(\epsilon \cos \left(\omega_{0} t+\delta\right), \epsilon \sin \left(\omega_{0} t+\delta\right), \sqrt{1-\epsilon^{2}}\right) .
$$

A polarized beam of electrons shot through such a film must exhibit Bloch spin resonance; but, in this experiment, it would be due to the rotation of the exchange field.

One might also envisage a Stern Gerlach experiment for electrons. One would start by constructing a sandwich of two ferromagnetic metals, I (e.g. Fe) and II (e.g. Ni), with exchange fields $\mathbf{W}_{I}$ and $\mathbf{W}_{I I}$ of different strength, joined by a transition region, a mixture of I and II, of width $d_{0}$. The transition region would be parallel to the $x y$-plane (see Fig. 2). One shoots an unpolarized beam of (not very 


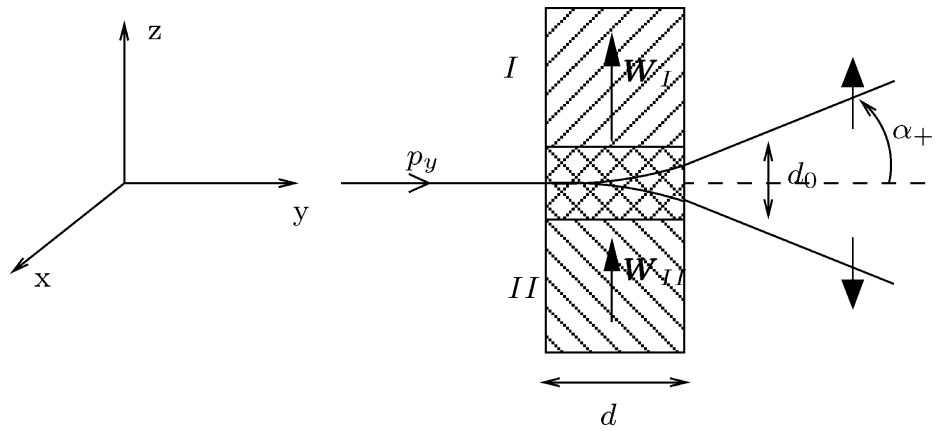

Fig. 2. Stern-Gerlach experiment.

hot) electrons through the sandwich along the transition region between I and II, as shown in Fig. 2. One would expect to detect two beams emerging on the other side of the film in slightly different directions that are spin-polarized in opposite directions.

The force, $f$, in the $z$-direction on an electron with spin up/down inside the film is given, approximately, by

$$
f \cong \pm \frac{\left|\mathbf{W}_{I}-\mathbf{W}_{I I}\right|}{2 d_{0}}
$$

It yields a change in the $z$-component of the momentum of the electron during its passage through the film given by

$$
\Delta p_{z} \cong \pm \frac{\left|\mathbf{W}_{I}-\mathbf{W}_{I I}\right|}{2 d_{0}} \frac{d}{v},
$$

where $v$ is the average group velocity. The deflection angle $\alpha$ is then found from

$$
\tan \alpha \cong \frac{\Delta p_{z}}{p_{y}} .
$$

Of course, as discussed above, the intensity, $I_{+}$of the upper beam can be expected to be much larger than the intensity, $I_{-}$, of the lower beam, due to spin-asymmetric absorption inside the film.

In an experimental set-up similar to the one above, one could force an electron current, spin-polarized in the $z$-direction, through the film. Then a Hall tension in the $z$-direction should be observed (Hall effect for spin currents). Generally speaking the Hall tension is parallel to the gradient of the Weiss exchange field, while the Hall current is perpendicular to it. This and other related effects are discussed in some detail in Ref. 14. 


\section{THE WEISS EXCHANGE FIELD IN GENERAL ONE-BAND $(t-J$ AND HUBBARD) TIGHT-BINDING MODELS}

In this section, we study the origin and dynamics of the Weiss exchange field in simple tight-binding models. We start by considering one-band models; but it is straightforward to include higher (partially occupied or empty) bands relevant for the study of electron transmission through magnetic films.

Every site, $x$, of a lattice $\Gamma \stackrel{\text { e.g. }}{=} \mathbb{Z}^{d}, d=1,2,3, \ldots$ carries a four-dimensional state space, $\mathbb{C}^{4}$, corresponding to an empty state, a one-electron state with spin up $(+)$ or down $(-)$, or a state of two electrons in a spin-singlet state. The corresponding basis vectors of $\mathbb{C}^{4}$ are denoted by $\left\{|0\rangle_{x},|+\rangle_{x},|-\rangle_{x},|+-\rangle_{x}\right\}$, $x \in \Gamma$. We introduce electron creation- and annihilation operators, $c_{s}^{\dagger}(x)$ and $c_{s}(x)$, respectively, where $s= \pm, x \in \Gamma$, satisfying canonical anti-commutation relations

$$
\left\{c_{s}^{\#}(x), c_{s^{\prime}}^{\#}\left(x^{\prime}\right)\right\}=0, \quad\left\{c_{s}^{\dagger}(x), c_{s^{\prime}}\left(x^{\prime}\right)\right\}=\delta_{s s^{\prime}} \delta_{x x^{\prime}},
$$

with $c^{\#}=c$ or $c^{\dagger}$. Then

$$
c_{s}(x)|0\rangle_{x}=0, \quad \text { for } s= \pm, x \in \Gamma,
$$

and

$$
|s\rangle_{x}=c_{s}^{\dagger}(x)|0\rangle_{x}, \quad \text { etc. }
$$

Number operators are given by

$$
n_{s}(x)=c_{s}^{\dagger}(x) c_{s}(x), \quad n(x)=n_{+}(x)+n_{-}(x),
$$

where $n_{s}(x)$ measures the number of electrons at site $x$ with spin $s$. By $(3.1), n_{s}(x)$ has eigenvalues 0 and 1 , while $n(x)$ has eigenvalues 0,1 and 2 . The spin operator, $\mathbf{S}(x)$, at site $x$ is given by

$$
\mathbf{S}(x)=\frac{1}{2} \sum_{s, s^{\prime}} c_{s}^{\dagger}(x) \boldsymbol{\sigma}_{s s^{\prime}} c_{s^{\prime}}(x),
$$

where $\sigma_{s s^{\prime}}$ is the $\left(s s^{\prime}\right)$-matrix element of the vector of Pauli matrices $\sigma=$ $\left(\sigma_{x}, \sigma_{y}, \sigma_{z}\right)$. The operators $\mathbf{S}(x)$ vanish on $|0\rangle_{x}$ and on $|+-\rangle_{x}$, while, for any linear combination, $\phi(x)$, of $|+\rangle_{x}$ and $|-\rangle_{x}$,

$$
\mathbf{S}(x) \cdot \mathbf{S}(x) \phi(x)=\frac{3}{4} \phi(x) .
$$

The dynamics of a gas of electrons moving on the lattice $\Gamma$ is assumed to be generated by a $t-J$ model Hamiltonian of the form

$$
H=T+U+E_{\neq},
$$


where the kinetic energy operator, $T$, (the hopping term) is given by

$$
\begin{aligned}
T & =\sum_{x \neq y} \hat{t}(x-y) \sum_{s} c_{s}^{\dagger}(x) c_{s}(y), \\
U & =U_{0} \sum_{x} n_{+}(x) n_{-}(x)
\end{aligned}
$$

describes an on-site (Hubbard) repulsion, and

$$
E_{\neq}=-\frac{1}{2} \sum_{x, y} J_{\neq}(x-y) \mathbf{S}(x) \cdot \mathbf{S}(y)
$$

describes (effective) exchange interactions between the spins of electrons. In (3.8), $\hat{t}(x-y)$ is the amplitude for hopping of an electron from site $y$ to site $x$. Selfadjointness of the Hamiltonian $H$ implies that $\hat{t}(x-y)=\overline{\hat{t}(y-x)}$. The constant $U_{0}$ is a measure of the strength of the on-site repulsion, and $J_{\neq}(x-y)$ is the exchange coupling between spins at $x$ and $y$, with $J_{\neq}(0)=0$.

The term $U$ can be rewritten as follows. When $x$ is empty or singly occupied $U_{0} n_{+}(x) n_{-}(x)$ vanishes; when $x$ is doubly occupied it takes the value $U_{0}$. Thus, by (3.6),

$$
U_{0} n_{+}(x) n_{-}(x)=\frac{1}{2} U_{0} n(x)-\frac{2}{3} U_{0} \mathbf{S}(x) \cdot \mathbf{S}(x)=-\frac{2}{3} U_{0}: \mathbf{S}(x) \cdot \mathbf{S}(x):,
$$

where : . : denotes normal ordering. We now set $J(0)=\frac{4}{3} U_{0}, J(x)=J_{\neq}(x)$, for $x \neq 0$, and find

$$
H=T+E,
$$

with

$$
E=-\frac{1}{2} \sum_{x, y} J(x-y): \mathbf{S}(x) \cdot \mathbf{S}(y): .
$$

The Heisenberg model corresponds to a completely flat band, i.e.,

$$
T=0, \quad \text { or } \hat{t}(x) \equiv 0 .
$$

Our goal is to study the grand-canonical partition function

$$
\Xi(\beta, \mu):=\operatorname{Tr}\left(e^{-\beta\left[H-\mu \sum_{x} n(x)\right]}\right)
$$

and the thermal equilibrium state given by the density matrix

$$
P_{\beta, \mu}=\Xi(\beta, \mu)^{-1} e^{-\beta\left[H-\mu \sum_{x} n(x)\right]}
$$

for the family of Hamiltonians introduced above. 
Remark: Of course, these objects must first be calculated for arbitrary bounded regions, $\Lambda$, in the lattice $\Gamma$ (indicated by a superscript " $\Lambda$ "). Afterwards, one may attempt to pass to the thermodynamic limit, $\Lambda \nearrow \Gamma$, setting

$$
\beta g(\beta, \mu)=-\lim _{\Lambda \nearrow \Gamma} \frac{1}{|\Lambda|} \ln \Xi^{\Lambda}(\beta, \mu)
$$

and

$$
\omega_{\beta, \mu}(\cdot)=\lim _{\Lambda \nearrow \Gamma} \operatorname{Tr}\left(P_{\beta, \mu}^{\Lambda}(\cdot)\right),
$$

where $|\Lambda|$ is the number of sites in $\Lambda$. These are standard matters (see e.g. Ref. 27) and will not be discussed any further. The superscript " $\Lambda$ " will often be suppressed in our notation.

It is convenient to study $\Xi(\beta, \mu)$ and the state $\omega_{\beta, \mu}$ by making use of functional integration. With every $x \in \Gamma$ and every imaginary time $t \in[0, \beta)$, we associate anticommuting (Grassmann) variables, $\bar{C}_{s}(x, t)$ and $C_{s}(x, t)$, with

$$
\left\{C_{s}^{(-)}(x, t), \stackrel{(-)}{C_{s^{\prime}}}\left(x^{\prime}, t^{\prime}\right)\right\}=0,
$$

and

$$
C_{s}^{\#}(x, t+\beta)=-C_{s}^{\#}(x, t) .
$$

The anti-periodic boundary conditions in (3.19) are a consequence of the KMS condition; see e.g. Ref. 17. We introduce an (imaginary-time) action functional

$$
\begin{aligned}
S_{J}(\bar{C}, C)= & \int_{0}^{\beta} d t\left[\left(\sum_{x, s} \bar{C}_{s}(x, t) \frac{\partial}{\partial t} C_{s}(x, t)\right)-H(\bar{C}(., t), C(., t))\right. \\
& \left.-\mu \sum_{x, s} \bar{C}_{s}(x, t) C_{s}(x, t)\right],
\end{aligned}
$$

where $H(\bar{C}, C)$ is obtained from the Hamiltonian $H$ by replacing the operators $c_{s}(x)$ and $c_{s}^{\dagger}(x)$ by $C_{s}(x, t)$ and $\bar{C}_{s}(x, t)$, respectively. Because of the presence of products of $C$ - and $\bar{C}$-fields at coinciding sites and imaginary times the quartic term needs to be regularized; (the ambiguity of the quadratic terms is an unimportant constant). We introduce an infinitesimal separation of the imaginary times, setting

$$
\mathbf{S}^{\epsilon}(x, t)=\frac{1}{2} \sum_{s, s^{\prime}} \bar{C}_{s}(x, t+\epsilon) \boldsymbol{\sigma}_{s s^{\prime}} C_{s^{\prime}}(x, t-\epsilon)
$$

in the action. Then the time ordering of the $C$ - and $\bar{C}$-fields corresponds to the normal ordering of the operators $c^{\sharp}$. Using (3.13), the regularized action is seen to 
be given by

$$
\begin{aligned}
S_{J}^{\epsilon}(\bar{C}, C)= & \int_{0}^{\beta} d t\left[\left(\sum_{x, s} \bar{C}_{s}(x, t) \frac{\partial}{\partial t} C_{s}(x, t)\right)\right. \\
& -\sum_{x \neq y} \hat{t}(x-y) \sum_{s} \bar{C}_{s}(x, t) C_{s}(y, t)+\frac{1}{2} \sum_{x, y} J(x-y) \\
& \left.\times \mathbf{S}^{\epsilon}(x, t) \cdot \mathbf{S}^{\epsilon}(y, t)+\mu \sum_{x, s} \bar{C}_{s}(x, t) C_{s}(x, t)\right],
\end{aligned}
$$

Berezin's integration form for anticommuting variables is formally given by

$$
\mathcal{D} \bar{C} \mathcal{D} C=\Pi_{t \in[0, \beta)} \Pi_{x} \Pi_{s} d \bar{C}_{s}(x, t) d C_{s}(x, t) .
$$

Then

$$
\Xi(\beta, \mu)=\lim _{\epsilon \searrow 0} \int \mathcal{D} \bar{C} \mathcal{D} C e^{S_{J}^{\epsilon}(\bar{C}, C)}
$$

and the state $\omega_{\beta, \mu}$ can be reconstructed from the imaginary-time Green functions

$$
\begin{aligned}
\left\langle\Pi_{j} \bar{C}_{s_{j}}\left(x_{j}, t_{j}\right) C_{s_{j}^{\prime}}\left(x_{j}^{\prime}, t_{j}^{\prime}\right)\right\rangle_{\beta, \mu}= & \Xi(\beta, \mu)^{-1} \lim _{\epsilon \searrow 0} \int \mathcal{D} \bar{C} \mathcal{D} C e^{S_{J}^{\epsilon}(\bar{C}, C)} \\
& \times \Pi_{j} \bar{C}_{s_{j}}\left(x_{j}, t_{j}\right) C_{s_{j}^{\prime}}\left(x_{j}^{\prime}, t_{j}^{\prime}\right),
\end{aligned}
$$

see, e.g. Refs. 17, 28.

If $S_{J}^{\epsilon}(\bar{C}, C)$ were quadratic in $C$ and $\bar{C}$ the Berezin integrals in (3.24), (3.25) could be evaluated and expressed in terms of determinants. The only contribution to $S_{J}^{\epsilon}(\bar{C}, C)$ not quadratic in $\bar{C}, C$ comes from the term

$$
\int_{0}^{\beta} d t \sum_{x, y} J(x-y) \mathbf{S}^{\epsilon}(x, t) \cdot \mathbf{S}^{\epsilon}(y, t) .
$$

By introducing a Lagrange-multiplier field ("Hubbard-Stratonovich transformation") this term, too, can be rendered quadratic in $\bar{C}$ and $C$. The Lagrange multiplier field will turn out to be the Weiss exchange field $\mathbf{W}$.

Let $K$ denote the matrix inverse of $J$, i.e., $\hat{K}(\mathbf{k})=\hat{J}(\mathbf{k})^{-1}$, where $\mathbf{k}$ is a point in the first Brillouin zone, $B_{\Gamma}$, of the lattice $\Gamma$, and $\hat{J}, \hat{K}$ denote the Fourier transforms of $J$ and $K$. If $U_{0}$ is chosen to be large enough then $J$ and $K$ are positivedefinite, i.e., $\hat{J}(\mathbf{k})>0, \hat{K}(\mathbf{k})>0$, for $\mathbf{k} \in B_{\Gamma}$. We introduce a real, vector-valued random field, $\mathbf{W}(x, t), x \in \Gamma, t \in[0, \beta)$, with

$$
\mathbf{W}(x, t+\beta)=\mathbf{W}(x, t)
$$


(KMS condition for bosons; see e.g. Ref. 17), assumed to have a Gaussian distribution

$$
d \mu_{K}(\mathbf{W})=e^{S_{K}(\mathbf{W})} \mathcal{D} \mathbf{W},
$$

where

$$
\begin{gathered}
S_{K}(\mathbf{W})=-\frac{1}{2} \int_{0}^{\beta} d t \sum_{x, y} \mathbf{W}(x, t) K(x-y) \mathbf{W}(y, t), \\
\mathcal{D} \mathbf{W}=\operatorname{const}_{K} \Pi_{t \in[0, \beta)} \Pi_{x} d^{3} W(x, t),
\end{gathered}
$$

and const $_{K}$ is chosen such that

$$
\int d \mu_{K}(\mathbf{W})=1
$$

We define ${ }^{6}$

$$
\begin{gathered}
S_{0}(\bar{C}, C)=S_{J=0}(\bar{C}, C), \\
S_{1}(\bar{C}, C ; \mathbf{W})=-\int_{0}^{\beta} d t\left(\sum_{x} \mathbf{W}(x, t) \cdot \mathbf{S}(x, t)\right),
\end{gathered}
$$

and

$$
\bar{S}(\bar{C}, C ; \mathbf{W})=S_{0}(\bar{C}, C)+S_{1}(\bar{C}, C ; \mathbf{W})+S_{K}(\mathbf{W}) .
$$

We note that $\bar{S}(\bar{C}, C ; \mathbf{W})$ is quadratic in $\bar{C}$ and $C$ and in $\mathbf{W}$ (separately), and that

$$
\int \mathcal{D} \mathbf{W} e^{\bar{S}(\bar{C}, C ; \mathbf{W})}=e^{S_{J}(\bar{C}, C)},
$$

as follows by quadratic completion.

It is apparent from (3.33) that $\mathbf{W}(x, t)$ plays the role of the Weiss exchange field. In order to calculate the grand canonical partition function $\Xi(\beta, \mu)$, we have to integrate the R.S. of (3.35) over $\bar{C}$ and $C$. Since $\bar{S}(\bar{C}, C ; \mathbf{W})$ is quadratic in $\bar{C}$ and $C$, it is tempting to interchange the $\mathbf{W}-$ and the $\bar{C}, C$ - integrations. First doing the $\bar{C}, C$-integration yields

$$
\int \mathcal{D} \bar{C} \mathcal{D} C e^{S_{0}(\bar{C}, C)+S_{1}(\bar{C}, C ; \mathbf{W})}=\operatorname{det}\left(D_{\mathbf{W}}\right),
$$

where the operator $D_{\mathbf{W}}$ acts on the space

$$
\mathfrak{h}=C^{\infty}([0, \beta))_{a p} \otimes\left(l^{2}(\Gamma) \otimes \mathbb{C}^{2}\right)
$$

$\overline{{ }^{6} \text { Henceforth }}$ we suppress the regularization indicated by $\epsilon$. 
("ap" stands for "antiperiodic") and is determined by requiring that

$$
S_{0}(\bar{C}, C)+S_{1}(\bar{C}, C ; \mathbf{W})=\int_{0}^{\beta} d t \sum_{x, s} \bar{C}(x, t)\left(D_{\mathbf{W}} C\right)(x, t) .
$$

Thus, $D_{\mathbf{W}}$ is given by

$$
D_{\mathbf{W}}=\frac{\partial}{\partial t} \otimes \mathbb{1}+\mathbb{1} \otimes(\hat{t}-\mu 1)+\frac{1}{2} \mathbf{W}(x, t) \cdot \boldsymbol{\sigma},
$$

where $(\hat{t} h)(x)=\sum_{y} \hat{t}(x-y) h(y), \quad h \in l^{2}(\Gamma)$.

After Fourier transformation in $t$ and $x$, vectors in the space $\mathfrak{h}$ are given by two-component spinors

$$
\phi\left(\mathbf{k}, k_{0}\right)=\left(\begin{array}{c}
f_{+}\left(\mathbf{k}, k_{0}\right) \\
f_{-}\left(\mathbf{k}, k_{0}\right)
\end{array}\right),
$$

with $\mathbf{k} \in B_{\Gamma}$ and $k_{0}=(\pi / \beta)(2 n+1), n \in \mathbb{Z}$, and

$$
\begin{aligned}
\left(D_{\mathbf{W}} \phi\right)\left(\mathbf{k}, k_{0}\right)= & \left(-i k_{0}+t(\mathbf{k})-\mu\right) \phi\left(\mathbf{k}, k_{0}\right)+\frac{1}{2} \int_{B_{\Gamma}} \frac{d \mathbf{k}^{\prime}}{(2 \pi)^{3}} \\
& \times \sum_{k_{0}^{\prime}} \hat{\mathbf{W}}\left(\mathbf{k}-\mathbf{k}^{\prime}, k_{0}-k_{0}^{\prime}\right) \cdot(\sigma \phi)\left(\mathbf{k}^{\prime}, k_{0}^{\prime}\right),
\end{aligned}
$$

where $t(\mathbf{k})$ is the Fourier transform of $\hat{t}(x)$. Defining the effective action, $S_{\text {eff }}(\mathbf{W})$, of the exchange field, $\mathbf{W}$, by

$$
S_{\text {eff }}(\mathbf{W})=\ln \operatorname{det}\left(D_{\mathbf{W}}\right)+S_{K}(\mathbf{W}),
$$

we find that, for example,

$$
\Xi(\beta, \mu)=\int \mathcal{D} \mathbf{W} e^{S_{\text {eff }}(\mathbf{W})} .
$$

Note that

$$
\frac{\delta}{\delta \mathbf{W}(x, t)} e^{\bar{S}(\bar{C}, C ; \mathbf{W})}=-\left(\sum_{y} K(x-y) \mathbf{W}(y, t)+\mathbf{S}(x, t)\right) e^{\bar{S}(\bar{C}, C ; \mathbf{W})} .
$$

Setting

$$
\delta_{\mathbf{W}(x, t)}=\frac{\delta}{\delta \mathbf{W}(x, t)}+\sum_{y} K(x-y) \mathbf{W}(y, t),
$$


the imaginary-time Green function corresponding to a product, $\prod_{j=1}^{n} S_{\alpha_{j}}\left(x_{j}, t_{j}\right)$, of spin operators turns out to be given by

$$
\left\langle\prod_{j=1}^{n} S_{\alpha_{j}}\left(x_{j}, t_{j}\right)\right\rangle_{\beta, \mu}=(-1)^{n} \Xi(\beta, \mu)^{-1} \int \mathcal{D} \mathbf{W} \prod_{j=1}^{n} \delta_{W_{\alpha_{j}}\left(x_{j}, t_{j}\right)} e^{S_{\mathrm{eff}}(\mathbf{W})},
$$

where we have used (3.36), (3.40) and (3.42).

Our purpose is now to determine $S_{\text {eff }}(\mathbf{W})$ as explicitly as possible, in order to get some insight into the R.S. of (3.41) and (3.43); see also Ref. 29.

\section{EFFECTIVE $\sigma$-MODELS OF THE EXCHANGE FIELD}

If we want to apply the method of steepest descent to estimate the R.S. of (3.41) and of (3.43), we must look for the (absolute and local) maxima of $S_{\text {eff }}(\mathbf{W})$. This leads us to consider mean-field ansätze for configurations, $\mathbf{W}$, corresponding to local maxima of $S_{\text {eff }}(\mathbf{W})$.

\subsection{Ferromagnetic Mean-Field Theory}

We set

$$
\mathbf{W}(x, t)=W_{0} \mathbf{n},
$$

independently of $x$ and $t$, where $\mathbf{n}$ is a unit vector, and $W_{0}>0$. Because of the symmetry of the problem under rotations of $\mathbf{W}$, we can choose $\mathbf{n}=\mathbf{n}_{z}$ to point into the $z$-direction. The problem of evaluating

$$
g^{m f}\left(\beta, \mu ; W_{0}\right)=\lim _{\Lambda \nearrow \Gamma}-\frac{1}{\beta|\Lambda|} S_{\text {eff }}^{\Lambda}\left(\mathbf{W} \equiv W_{0} \mathbf{n}_{z}\right)
$$

is elementary, because, in momentum space, all modes decouple from one another, and we can appeal to well known calculations (see e.g. Refs. 15, 17) to obtain the answer:

$$
\begin{aligned}
g^{m f}\left(\beta, \mu ; W_{0}\right)= & \frac{1}{2} \hat{K}(0) W_{0}^{2}-\lim _{\Lambda \nearrow \Gamma} \frac{1}{\beta|\Lambda|} \ln \operatorname{det}\left(D_{\mathbf{W}=W_{0} \mathbf{n}}\right) \\
= & \frac{1}{2} \hat{K}(0) W_{0}^{2}-\frac{1}{\beta} \int_{B_{\Gamma}} \frac{d \mathbf{k}}{(2 \pi)^{3}} \ln \left[\left(1+e^{-\beta\left(\epsilon(\mathbf{k})+W_{0} / 2\right)}\right)\right. \\
& \left.\times\left(1+e^{-\beta\left(\epsilon(\mathbf{k})-W_{0} / 2\right)}\right)\right]+ \text { const. },
\end{aligned}
$$

where

$$
\epsilon(\mathbf{k})=t(\mathbf{k})-\mu .
$$


Formula(4.3) can also be derived directly from the definition of $\ln \operatorname{det}\left(D_{\mathbf{W}}\right)=$ $\operatorname{Tr} \ln \left(D_{\mathbf{W}}\right)$ by using the Poisson summation formula.

In the limit $\beta \longrightarrow \infty$, expression (4.3) converges to

$$
\begin{aligned}
g^{m f}\left(\infty, \mu ; W_{0}\right)= & \frac{1}{2} \hat{K}(0) W_{0}^{2} \\
& +\int_{B_{\Gamma}} \frac{d \mathbf{k}}{(2 \pi)^{3}}\left[2 \epsilon(\mathbf{k}) \Theta\left(-\frac{W_{0}}{2}-\epsilon(\mathbf{k})\right)+\left(\epsilon(\mathbf{k})-\frac{W_{0}}{2}\right)\right. \\
& \left.\Theta\left(\frac{W_{0}}{2}-|\epsilon(\mathbf{k})|\right)\right]+ \text { const. }
\end{aligned}
$$

which is the ground state energy density of electrons in a constant exchange field $\mathbf{W}=W_{0} \mathbf{n}_{z}$. The value of $W_{0}$ minimizing $g^{m f}$ is found by solving the equation $\left(\partial / \partial W_{0}\right) g^{m f}\left(\beta, \mu ; W_{0}\right)=0$, i.e.,

$$
\hat{K}(0) W_{0}=\frac{1}{2} \int_{B_{\Gamma}} \frac{d \mathbf{k}}{(2 \pi)^{3}}\left[\left(e^{\beta\left(\epsilon(\mathbf{k})-\frac{W_{0}}{2}\right)}+1\right)^{-1}-\left(e^{\beta\left(\epsilon(\mathbf{k})+\frac{W_{0}}{2}\right)}+1\right)^{-1}\right] .
$$

When $\beta \rightarrow 0$ the R.S. of (4.6) tends to 0 , and we conclude that, for small $\beta$, this equation only has the trivial solution, $W_{0}=0$. When $\beta \rightarrow \infty$, Eq. (4.6) yields the equation

$$
\hat{K}(0) W_{0}=\frac{1}{2} \int_{B_{\Gamma}} \frac{d \mathbf{k}}{(2 \pi)^{3}} \Theta\left(\frac{W_{0}}{2}-|\epsilon(\mathbf{k})|\right) .
$$

Besides the trivial solution, this equation also has a non-trivial solution $W_{0}>0$, provided $\hat{K}(0)$ is sufficiently small (depending on $\hat{t}$ ). We recall that

$$
\hat{K}(0)=\hat{J}(0)^{-1}=\left(\frac{4 U_{0}}{3}+\hat{J}_{\neq}(0)\right)^{-1} .
$$

Thus, for $U_{0} / t_{*}$, with $t_{*}:=\max |\hat{t}(x)|$, large enough, Eq. (4.6) has a non-trivial solution, provided $\beta$ is large enough, even in the Hubbard model, where $J_{\neq}=0$. Note that, by (3.42) and (3.43),

$$
-\sum_{y} K(x-y)\langle\mathbf{W}(y, t)\rangle_{\beta, \mu}=\langle\mathbf{S}(x, t)\rangle_{\beta, \mu} .
$$

Thus,

$$
\hat{K}(0) W_{0}=M,
$$

where $W_{0}$ is the non-trivial solution of Eq. (4.6), and $M$ is the spontaneous magnetization in mean-field theory (i.e., $-M \mathbf{n}_{z}=\langle\mathbf{S}(x, t)\rangle_{\beta, \mu}^{m f}$ ). Thus, Eq. (4.7) 


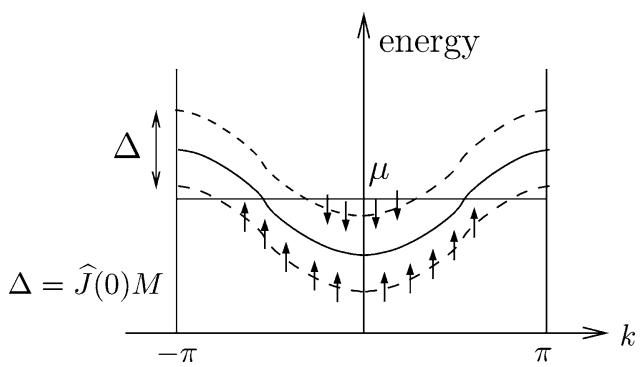

Fig. 3. Spin-split bands.

tells us that, at zero temperature $(\beta \rightarrow \infty)$,

$$
M=\frac{1}{2} \int_{B_{\Gamma}} \frac{d \mathbf{k}}{(2 \pi)^{3}} \Theta\left(\frac{\hat{J}(0)}{2} M-|\epsilon(\mathbf{k})|\right),
$$

where $\hat{J}(0)=\sum_{x} J(x)=\hat{K}(0)^{-1}$. This equation has an obvious interpretation apparent from Fig. 3.

Matters simplify further for a flat band, $t \equiv 0$, i.e., for the Heisenberg model. Then eq. (4.7) reduces to

$$
M=\left\{\begin{array}{l}
1 / 2, \quad-\hat{J}(0) / 4<\mu<\hat{J}(0) / 4, \\
0, \quad \text { otherwise },
\end{array}\right.
$$

for $\beta \rightarrow \infty$. For, Eq. (4.5) implies that

$$
g^{m f}\left(\infty, \mu ; W_{0}\right)=\frac{1}{2} \hat{K}(0) W_{0}^{2}-\frac{W_{0}}{2} \Theta\left(\frac{W_{0}}{2}-|\mu|\right),
$$

up to an unimportant constant. Thus, for $|\mu|<1 /(4 \hat{K}(0))=\hat{J}(0) / 4$, $g^{m f}\left(\infty, \mu ; W_{0}\right)$ has a quadratic minimum at $W_{0}=1 /(2 \hat{K}(0))$, with

$$
g^{m f}\left(\infty, \mu ; W_{0}=\frac{1}{2 \hat{K}(0)}\right)=-\frac{1}{2 \hat{K}(0)},
$$

and

$$
\frac{\partial^{2} g^{m f}}{\partial W_{0}^{2}}\left(\infty, \mu ; W_{0} \approx \frac{1}{2 \hat{K}(0)}\right) \equiv \hat{K}(0) .
$$

\subsection{Antiferromagnetic Mean-Field Theory}

Antiferromagnetism and Néel ordering in Hubbard models is discussed in some detail in Ref. 15, so we shall be brief. To simplify matters, we consider 
the lattices $\Gamma=\mathbb{Z}^{d}, d=1,2,3, \ldots$ Our mean-field ansatz configuration for the exchange field $\mathbf{W}$ is given by

$$
\mathbf{W}(x, t)=(-1)^{|x|} W_{0} \mathbf{n}_{z},
$$

which describes Néel order. We skip detailed calculations and give the result for the free energy

$$
\begin{aligned}
g^{m f}\left(\beta, \mu, W_{0}\right)=\frac{1}{2} & \hat{K}\left(\mathbf{k}_{\pi}\right) W_{0}^{2}-\frac{1}{\beta} \int_{B_{\Gamma}} \frac{d \mathbf{k}}{(2 \pi)^{3}} \ln \left[\left(1+e^{-\beta \sqrt{\epsilon(\mathbf{k})^{2}+(1 / 4) W_{0}^{2}}}\right)\right. \\
& \left.\times\left(1+e^{\beta \sqrt{\epsilon(\mathbf{k})^{2}+(1 / 4) W_{0}^{2}}}\right)\right]+ \text { const },
\end{aligned}
$$

where $\mathbf{k}_{\pi}=(\pi, \pi, \ldots, \pi)$ denotes a corner of the Brillouin zone. In order to derive (4.16), we assume that $\epsilon\left(\mathbf{k}+\mathbf{k}_{\pi}\right)=-\epsilon(\mathbf{k})$, i.e., we assume the band to be half-filled. Note that small deviations, $\delta \epsilon(\mathbf{k})=\epsilon\left(\mathbf{k}+\mathbf{k}_{\pi}\right)+\epsilon(\mathbf{k})$, from half filling can be taken into account with the help of perturbation theory in $\delta \epsilon(\mathbf{k})$. The idea behind the derivation of (4.16) is to consider simultaneously the contributions of $\mathbf{k}$ and $\mathbf{k}+\mathbf{k}_{\pi}$, and then diagonalise a $4 \times 4$ matrix. The energy eigenvalues, for fixed $\mathbf{k}$, are given by $\epsilon_{ \pm}(\mathbf{k})= \pm \sqrt{\epsilon^{2}(\mathbf{k})+(1 / 4) W_{0}^{2}}$, each one with twofold degeneracy. From (4.16) we obtain the mean-field value of $W_{0}$ by solving the equation $\partial g^{m f}\left(\beta, \mu ; W_{0}\right) / \partial W_{0}=0$. In the limit where $\beta \rightarrow \infty$, this equation reduces to

$$
\hat{K}\left(\mathbf{k}_{\pi}\right)=\frac{1}{4} \int_{B_{\Gamma}} \frac{d \mathbf{k}}{(2 \pi)^{3}} \frac{1}{\sqrt{\epsilon(\mathbf{k})^{2}+(1 / 4) W_{0}^{2}}} .
$$

The R.S. diverges logarithmically when $W_{0} \rightarrow 0$, for a lattice $\Gamma$ of arbitrary dimension. Thus (4.17) has a non-trivial solution $W_{0}>0$ for arbitrarily large values of $\hat{K}\left(\mathbf{k}_{\pi}\right)=\left(4 U_{0} / 3+J_{\neq}\left(\mathbf{k}_{\pi}\right)\right)^{-1}$, i.e., for arbitrarily small values of the on-site repulsion $U_{0}$ (even if $J_{\neq}=0$ ), and hence the same is true for finite, but sufficiently large values of $\beta$. Thus

$$
\left|\epsilon_{ \pm}(\mathbf{k})\right| \geq \frac{1}{2} W_{0}>0,
$$

and we conclude that a strictly positive energy gap $W_{0}>0$ opens at the Fermisurface.

We note that the solution of (4.17) behaves like

$$
W_{0} \propto t_{*} e^{-\alpha \hat{K}\left(\mathbf{k}_{\pi}\right) t_{*}},
$$

for some constant $\alpha$. For the half-filled Hubbard model $\left(J_{\neq}=0, U_{0}>0\right), \hat{K}\left(\mathbf{k}_{\pi}\right)=$ $3 /\left(4 U_{0}\right)$, and we find that $W_{0}$ has an essential singularity at $U_{0}=0$.

Comparing these results with those obtained for the ferromagnetic meanfield ansatz, we conclude that, at zero temperature, in the Hubbard model with 
$\hat{K}(0)=\hat{K}\left(\mathbf{k}_{\pi}\right)=3 /\left(4 U_{0}\right)$, a non-trivial ferromagnetic mean-field solution does not exist when $U_{0}$ is small, while an antiferromagnetic mean-field solution exists for arbitrarily small values of $U_{0}$ at half filling, where the (mean-field) ground state energy of the Néel state is found to be below the ground state energy of the ferromagnetic state.

\subsection{The Magnetic $\sigma$-Model}

As we have seen, the mean-field ansätze correspond to quadratic (local) maxima of the effective action as a function of the length $W_{0}$ of the exchange field. This suggests that the effective action $S_{\text {eff }}(\mathbf{W})$ is maximal on exchange-field configurations of constant length, $|\mathbf{W}(x, t)| \approx W_{0}$. It may therefore be appropriate to study the effective action (3.40) with the constraint

$$
|\mathbf{W}(x, t)|=W_{0}, \quad \text { for all } t \in[0, \beta), \quad x \in \Gamma .
$$

To study magnetic order, we consider the following ansatz for the effective action on configurations of exchange fields of constant length, $|\mathbf{W}(x, t)|=W_{0}$ :

$$
\begin{aligned}
\tilde{S}_{\mathrm{eff}}(\mathbf{W})= & \int_{0}^{\beta} d t \sum_{x}\left\{-\frac{W_{0}^{2}}{2} \sum_{y} \hat{\mathbf{W}}(x, t) K(x-y) \hat{\mathbf{W}}(y, t)\right. \\
& \left.+C_{t}\left|\partial_{t} \hat{\mathbf{W}}(x, t)\right|^{2}+C_{\nabla}|\nabla \hat{\mathbf{W}}(x, t)|^{2}\right\} \\
& +C_{W Z} \int_{S^{2, N}} d t d s \sum_{x} \hat{\mathbf{W}}(x, t, s) \cdot\left(\partial_{t} \hat{\mathbf{W}}(x, t, s) \wedge \partial_{s} \hat{\mathbf{W}}(x, t, s)\right) \\
& + \text { higher order terms },
\end{aligned}
$$

where we have set $\hat{\mathbf{W}}(x, t):=W_{0}^{-1} \mathbf{W}(x, t)$ and where, for each $x \in \Gamma, \hat{\mathbf{W}}(x, t, s)$ is chosen to be a smooth extension of $\hat{\mathbf{W}}(x, t)$ from the circle $\{t \mid 0 \leq t \leq \beta\}$ to the northern hemisphere, $S^{2, N}$, of the 2 -sphere of radius $\beta /(2 \pi)$. The operator $\nabla$ denotes the finite difference gradient on the lattice, and the corresponding term in the effective action has to be interpreted as

$$
\sum_{x}|\nabla \hat{\mathbf{W}}(x, t)|^{2}:=\sum_{|x-y|=1}|\hat{\mathbf{W}}(y, t)-\hat{\mathbf{W}}(x, t)|^{2} .
$$

The functional $\tilde{S}_{\text {eff }}(\mathbf{W})$ is the (imaginary-time) action of a non-linear $\sigma$-model with $S O(3)$ symmetry and with a Wess-Zumino term. This model is called magnetic $\sigma$-model. It is expected to describe exchange field configurations $\mathbf{W}$ in thermal equilibrium at inverse temperature $\beta$, provided the coefficients $C_{t}, C_{\nabla}$ and $C_{W Z}$ 
are chosen in such a way that

$$
\left.\tilde{S}_{\mathrm{eff}}(\mathbf{W}) \approx S_{\mathrm{eff}}(\mathbf{W})\right|_{|W(x, t)|=W_{0}}+\text { const } .
$$

on configurations $\mathbf{W}(x, t)$ satisfying the constraint $|\mathbf{W}(x, t)|=W_{0}$, with $S_{\text {eff }}(\mathbf{W})$ as in (3.40). More precisely, if $\mathbf{W}=\mathbf{W}_{m f}+\chi$, where $\mathbf{W}_{m f}$ is a mean(-exchange)field configuration and $\chi$ is a small perturbation of $\mathbf{W}_{m f}$, i.e., $\chi(x, t) \perp \mathbf{W}_{m f}(x)$ and $|\chi(x, t)| \ll 1$, for all $x \in \Gamma$ and all times $t$, then we want (4.22) to hold exactly to second order in $\chi$. Equation (4.21) makes it clear that, in order to calculate $C_{t}, C_{\nabla}$ and $C_{W Z}$ such that (4.22) holds to second order in $\chi$, it suffices to consider time-independent exchange fields, $\mathbf{W}(x, t) \equiv \mathbf{W}(x)$, to calculate $C_{\nabla}$, while it suffices to consider site-independent exchange fields, $\mathbf{W}(x, t) \equiv \mathbf{W}(t)$, when calculating $C_{t}$ and $C_{W Z}$. Obviously, the signs of $C_{t}$ and $C_{\nabla}$ will determine the stable mean-field configuration $\mathbf{W}_{m f}$. For $\mathbf{W}_{m f}$ to be independent of time $t, C_{t}$ must be negative (with our sign convention). For ferromagnetism to prevail, $C_{\nabla}$ must be negative.

In the next two subsections, the coefficients $C_{t}$ and $C_{\nabla}$ are determined in such a way that (4.22) holds to second order in $\chi$, for an appropriate choice of $\mathbf{W}_{m f}$ (depending on our choice of parameters in the original model). The coefficient $C_{W Z}$ of the Wess-Zumino term will be calculated and shown to be imaginary in the next to last subsection of Sec. 4. The Wess-Zumino term is crucial in arriving at the correct magnon dynamics for ferromagnetically ordered magnets.

\subsection{Stability of Antiferromagnetic Ordering at Half Filling}

In this subsection we calculate the coefficients $C_{t}$ and $C_{\nabla}$ of the magnetic $\sigma$-model (4.21), in such a way that (4.22) holds, in a regime where these coefficients can be calculated perturbatively. For the Hubbard model, this is the regime where the band is half-filled, and the signs of the coefficients $C_{t}$ and $C_{\nabla}$ will indeed determine the stable mean exchange field to be time-independent and antiferromagnetically ordered.

We first derive an innocent looking, but important identity, which will also be useful in the next section. We set

$$
D_{\mathbf{W}}^{-}=-\partial_{t}+\epsilon-W, \quad \epsilon=\hat{t}-\mu 1 .
$$

Equation (3.38) shows that

$$
D_{\mathbf{W}}^{T}=-\partial_{t}+\epsilon+\frac{1}{2}\left(W_{1} \sigma_{1}-W_{2} \sigma_{2}+W_{3} \sigma_{3}\right),
$$

where the superscript " $T$ " stands for transposition, (i.e., taking the adjoint, followed by complex conjugation). Hence

$$
\sigma_{2} D_{\mathbf{W}}^{T} \sigma_{2}=-\partial_{t}+\epsilon-W=D_{\mathbf{W}}^{-} .
$$


Furthermore,

$$
D_{\mathbf{W}}^{*}=-\partial_{t}+\epsilon+W=D_{-\mathbf{W}}^{-} .
$$

Since $\sigma_{2}=\sigma_{2}^{*}=\sigma_{2}^{-1}$, it follows from (4.24) that

$$
\operatorname{det}\left(D_{\mathbf{W}}\right)=\operatorname{det}\left(D_{\mathbf{W}}^{T}\right)=\operatorname{det}\left(\sigma_{2} D_{\mathbf{W}}^{T} \sigma_{2}\right)=\operatorname{det}\left(D_{\mathbf{W}}^{-}\right),
$$

and hence

$$
\overline{\operatorname{det}\left(D_{\mathbf{W}}\right)}=\operatorname{det}\left(D_{\mathbf{W}}^{*}\right)=\operatorname{det}\left(D_{-\mathbf{W}}^{-}\right)=\operatorname{det}\left(D_{-\mathbf{W}}\right) .
$$

This identity shows that the real part of the logarithm of the determinant of $D_{\mathbf{W}}$ is given by:

$$
\Re \ln \operatorname{det}\left(D_{\mathbf{W}}\right)=\frac{1}{2} \ln \operatorname{det}\left(D_{\mathbf{W}} D_{-\mathbf{W}}\right),
$$

with

$$
D_{\mathbf{W}} D_{-\mathbf{W}}=\left(\partial_{t}+\epsilon\right)^{2}-\frac{W_{0}^{2}}{4}-[\epsilon, W]-\left(\partial_{t} W\right) .
$$

We are tempted to treat $[\epsilon, W]+\left(\partial_{t} W\right)$ as a perturbation of

$$
\Lambda:=\left(\partial_{t}+\epsilon\right)^{2}-\frac{W_{0}^{2}}{4} \text {. }
$$

This is justified if $|\epsilon| \ll W_{0}$ and $\left|\partial_{t} W\right| \ll W_{0}$. In this regime, the band of electrons with spin parallel to the background field, $\mathbf{W}_{m f}$, is filled and the band of electrons with spin anti-parallel to $\mathbf{W}_{m f}$ is empty, i.e., the lattice is half-filled. At half-filling, we expect the Hubbard model to exhibit an antiferromagnetic phase at sufficiently low temperatures. We recall that $\ln d e t=\operatorname{tr} \ln$ and expand the logarithm up to quadratic order in $[\epsilon, W] / \Lambda$ and $\left(\partial_{t} W\right) / \Lambda$. Terms linear in $W$ vanish at critical points of the effective action; $\mathbf{W}$-independent terms are omitted. We are then left with

$$
\Re \ln \operatorname{det}\left(D_{\mathbf{W}}\right)=-\frac{1}{4} \operatorname{Tr}\left(\Lambda^{-1}\left([\epsilon, W]+\left(\partial_{t} W\right)\right) \Lambda^{-1}\left([\epsilon, W]+\left(\partial_{t} W\right)\right)\right)+\text { h.o. , }
$$

where h.o. stands for terms of higher order in $|\epsilon| / W_{0}$ or $\left|\partial_{t} W\right| / W_{0}$. Next, we pull $\Lambda^{-1}$ through $[\epsilon, W]+\partial_{t} W$ using the identity

$$
\left[\Lambda^{-1},[\epsilon, W]+\left(\partial_{t} W\right)\right]=-\Lambda^{-1}\left[\Lambda,[\epsilon, W]+\left(\partial_{t} W\right)\right] \Lambda^{-1},
$$

which shows that the commutator only contributes higher-order terms to (4.29). Mixed terms with one spatial and one time derivative of $W$ do not contribute to the trace. We thus arrive at

$$
\Re \ln \operatorname{det}\left(D_{\mathbf{W}}\right)=-\frac{1}{4} \operatorname{Tr}\left(\Lambda^{-2}\left([\epsilon, W]^{2}+\left(\partial_{t} W\right)^{2}\right)\right)+\text { h.o. . }
$$


Comparison with (4.21) then yields an expression for $C_{\nabla}$,

$$
\begin{aligned}
C_{\nabla}= & \frac{W_{0}^{2}}{8(2 \pi)^{3}} \int_{B_{\Gamma}} d \mathbf{k}|\nabla \epsilon(\mathbf{k})|^{2} \frac{1}{\beta} \\
& \times \sum_{k_{0}} \frac{1}{\left(\left(-i k_{0}+\epsilon(\mathbf{k})\right)^{2}-W_{0}^{2} / 4\right)^{2}} \stackrel{\beta W_{0} \rightarrow \infty}{\longrightarrow} \frac{1}{4(2 \pi)^{3} W_{0}} \int_{B_{\Gamma}} d \mathbf{k}|\nabla \epsilon(\mathbf{k})|^{2},
\end{aligned}
$$

which is positive. Thus the real part of the $\sigma$-model action (4.21) for the Hubbardmodel exhibits its maximum (w.r.t. time-independent exchange fields) on configurations for which

$$
\int_{0}^{\beta} d t \sum_{x}|\nabla \hat{\mathbf{W}}(x, t)|^{2}
$$

is maximal, and this is the case for a Néel ordered (staggered) exchange field.

From (4.31) we can also read off the $C_{t}$-coefficient in (4.21) to be given by

$$
C_{t}=-\frac{W_{0}^{2}}{8} \frac{1}{\beta} \sum_{k_{0}} \frac{1}{\left(\left(-i k_{0}+\epsilon(\mathbf{k})\right)^{2}-W_{0}^{2} / 4\right)^{2}} \stackrel{\beta W_{0} \rightarrow \infty}{\longrightarrow}-\frac{1}{4 W_{0}},
$$

which is manifestly negative. From these calculations and the mean field Eq. (4.17) we conclude that the Hubbard model at half filling exhibits a stable antiferromagnetic phase if the temperature is sufficiently low. Note that, for a one-band Hubbard model with $\epsilon_{*} / U_{0}$ small enough, where $\epsilon_{*}=\max _{\mathbf{k}}|\epsilon(\mathbf{k})|$, our perturbative calculation of the coefficients $\left(C_{t}, C_{\nabla}, C_{W Z}\right)$ of the leading terms in the effective action of the Weiss exchange field is meaningful beyond the mean-field approximation. It can be improved systematically.

\subsection{Linear Stability of the Ferromagnetic Mean-Field Solution}

In this subsection we analyze conditions that imply stability of ferromagnetically ordered states of the exchange field. In the Hubbard model, we expect ferromagnetism to prevail when the band is less than half-filled and fairly flat. This expectation agrees with ones based on alternative approximations; see e.g. Ref. 30. Since there is no energy gap at the Fermi surface, the operator $\Lambda$ defined in (4.28) has zeros, and the perturbative expansion of the last subsection breaks down for a less than half-filled band. We therefore have to resort to a linear stability analysis around a ferromagnetically ordered mean exchange field. We will see that the stability of the ferromagnetically ordered exchange field configuration is due to contributions close to the Fermi surface.

The stability of a ferromagnetically ordered exchange field w.r.t. $x$-independent fluctuations can be shown quite easily, since the operator $D_{\mathbf{W}}$ is diagonal in momentum space when $\mathbf{W}(x, t) \equiv \mathbf{W}(t)$ is $x$-independent. We can 
then write

$$
\lim _{\Lambda \nearrow \Gamma} \frac{1}{|\Lambda|} \ln \operatorname{det}\left(D_{\mathbf{W}}\right)=\int_{B_{\Gamma}} \frac{d \mathbf{p}}{(2 \pi)^{3}} \ln \operatorname{det}\left(\partial_{t}+\epsilon(\mathbf{p})+\frac{\mathbf{W}(t)}{2} \cdot \boldsymbol{\sigma}\right) .
$$

In the operator formalism outlined at the beginning of Sec. 3,

$$
\operatorname{det}\left(\partial_{t}+\epsilon(\mathbf{p})+\frac{\mathbf{W}(t)}{2} \cdot \boldsymbol{\sigma}\right)=\mathrm{const} \times \operatorname{Tr}_{\mathbb{C}^{4}} U_{\epsilon, \mathbf{W}}(\beta, 0),
$$

where

$$
\begin{aligned}
\partial_{t} U_{\epsilon, \mathbf{W}}(t, s) & =h_{\epsilon, \mathbf{W}}(t) U_{\epsilon, \mathbf{W}}(t, s), \\
U_{\epsilon, \mathbf{W}}(t, t) & =\mathbb{1}_{4},
\end{aligned}
$$

and

$$
h_{\epsilon, \mathbf{W}}(t)=\left(\begin{array}{cccc}
0 & 0 & 0 & 0 \\
0 & \epsilon(\mathbf{p}) \mathbb{1}_{2}+ & \frac{\mathbf{w}(t)}{2} \cdot \sigma & 0 \\
0 & & & 0 \\
0 & 0 & 0 & 2 \epsilon(\mathbf{p})
\end{array}\right) .
$$

The trace of the propagator $U_{\epsilon, \mathbf{w}}(\beta, 0)$ is

$$
\operatorname{Tr}_{\mathbb{C}^{4}} U_{\epsilon, \mathbf{W}}(\beta, 0)=1+e^{-\beta \epsilon(\mathbf{p})}\left(\operatorname{Tr}_{\mathbb{C}^{4}} U_{\epsilon=0, \mathbf{W}}(\beta, 0)-2\right)+e^{-2 \beta \epsilon(\mathbf{p})} .
$$

Using the Hölder-inequality for traces (see e.g. Ref. 21), we find that

$$
\left|\operatorname{Tr}_{\mathbb{C}^{4}}\left(U_{0, \mathbf{w}}(\beta, 0)\right)\right| \leq \operatorname{Tr}_{\mathbb{C}^{4}}\left(U_{0, \mathbf{W}=W_{0} \cdot \mathbf{n}}(\beta, 0)\right),
$$

for an arbitrary constant unit vector $\mathbf{n}$. From this inequality we can conclude, that $x$-independent fluctuations of an exchange field of constant length around a ferromagnetic mean-field configuration do not decrease the free energy and hence are not a source of instability.

Since the calculation is rather easy, we give an explicit formula for the coefficient $C_{t}$ in the action of the magnetic $\sigma$-model, for configurations of the exchange field close to a constant one (ferromagnetic ordering). Because of the stability of the ferromagnetic state against $x$-independent fluctuations we must find a negative sign for this coefficient. We use the Hölder inequality (4.36), in order to get an upper bound for the large- $\beta$ behaviour of the trace in (4.35) and find that, for large $\beta$, and up to a $\mathbf{W}$-independent constant,

$$
S_{\text {eff }}(\mathbf{W}) \approx\left(V_{F}^{-}-V_{F}^{+}\right) \ln \operatorname{det}\left(\partial_{t}+W\right)+S_{K}(\mathbf{W}),
$$

where $V_{F}^{ \pm}=\operatorname{Vol}\left\{\mathbf{p} \mid \epsilon(\mathbf{p}) \pm W_{0} / 2<0\right\}$. In order to calculate $C_{t}$, we take a look at the real part of $\ln \operatorname{det}\left(\partial_{t}+W\right)$,

$$
\Re \ln \operatorname{det}\left(\partial_{t}+W\right)=\frac{1}{2} \ln \operatorname{det}\left[\left(\partial_{t}+W\right)\left(-\partial_{t}+W\right)\right]=\frac{1}{2} \ln \operatorname{det}\left(\Lambda+\left(\partial_{t} W\right)\right),
$$


where $\Lambda:=-\partial_{t}^{2}+(1 / 4) W_{0}^{2}$. We treat $\left(\partial_{t} W\right)$ as a perturbation of $\Lambda$ and proceed in the same way as in the last subsection to arrive at

$$
\Re \ln \operatorname{det}\left(\partial_{t}+W\right)=-\frac{1}{4} \operatorname{tr}\left(\Lambda^{-2}\left(\partial_{t} W\right)^{2}\right)+\text { h.o. }
$$

where we omit $\mathbf{W}$-independent terms. This yields the result

$$
C_{t}=-\frac{W_{0}^{2}\left(V_{F}^{-}-V_{F}^{+}\right)}{8(2 \pi)^{3} \beta} \sum_{k_{0}} \frac{1}{\left(k_{0}^{2}+W_{0}^{2} / 4\right)^{2}} \stackrel{\beta W_{0} \rightarrow \infty}{\longrightarrow}-\frac{V_{F}^{-}-V_{F}^{+}}{4(2 \pi)^{3} W_{0}},
$$

which is indeed manifestly negative.

Next, we investigate the stability of a ferromagnetically ordered meanfield configuration w.r.t. time-independent, but $x$-dependent perturbations, which amounts to the calculation of the coefficient $C_{\nabla}$ in the action of the magnetic $\sigma$-model. This calculation is somewhat subtle. We perturb the ferromagnetic mean-field configuration $\mathbf{W}=W_{0} \mathbf{n}_{z}$ by time-independent fluctuations, $\chi$, and calculate their contribution to the real part of the effective action to second order in $|\chi| / W_{0}$. Since the effective action has a quadratic minimum w.r.t. the length of the exchange field, we can assume $\chi$ to be orthogonal to $\mathbf{n}_{z}$. Then (omitting $\chi$-independent terms)

$$
\begin{aligned}
\Re S_{\mathrm{eff}}\left(W_{0} \mathbf{n}_{z}+\chi\right)= & -\frac{1}{2} \sum_{x, y} \chi^{T}(x) K(x-y) \chi(y)-\frac{1}{4} \operatorname{tr}\left(\Lambda^{-1}\{\boldsymbol{\epsilon}, \chi\} \Lambda^{-1}\right. \\
& \times\{\boldsymbol{\epsilon}, \boldsymbol{\chi}\})+ \text { h.o. }
\end{aligned}
$$

where

$$
\begin{aligned}
& \Lambda=-\partial_{t}^{2}+\epsilon^{2}+\frac{W_{0}^{2}}{4}+W_{0} \epsilon \sigma_{3}, \\
& \chi=\frac{1}{2} \chi \cdot \sigma .
\end{aligned}
$$

In the second term, we only keep contributions quadratic in $|\mathbf{p}| / W_{0}$, where $\mathbf{p}$ is the momentum labelling the modes of $\chi$ (derivative expansion). Spin degrees of freedom and Matsubara frequencies can be summed over explicitly, and - after a straightforward but tedious calculation-we obtain a formula for the coefficient, $C_{\nabla}$, of the term

$$
\frac{\beta}{W_{0}^{2}} \sum_{x}|\nabla \chi(x)|^{2}
$$


appearing in the expansion of the second term on the R.S. of (4.39). For large values of $\beta$, it is given by

$$
\begin{aligned}
C_{\nabla} \approx(2 \pi)^{-3} & \int_{B_{\Gamma}} d \mathbf{k} \frac{|\nabla \epsilon(\mathbf{k})|^{2}}{4 W_{0}} \\
& \times\left\{-\Theta\left(\epsilon(\mathbf{k})-\frac{W_{0}}{2}\right) \frac{\beta W_{0}}{4} \frac{1}{\cosh \beta\left(\epsilon(\mathbf{k})-\frac{W_{0}}{2}\right)+1}\right. \\
& +\Theta\left(\frac{W_{0}}{2}-|\epsilon(\mathbf{k})|\right)\left[1-\frac{\beta W_{0}}{4}\left(\frac{1}{\cosh \beta\left(\epsilon(\mathbf{k})+\frac{W_{0}}{2}\right)+1}\right.\right. \\
& \left.\left.+\frac{1}{\cosh \beta\left(\epsilon(\mathbf{k})-\frac{W_{0}}{2}\right)+1}\right)\right] \\
& \left.-\Theta\left(-\epsilon(\mathbf{k})-\frac{W_{0}}{2}\right) \frac{\beta W_{0}}{4} \frac{1}{\cosh \beta\left(\epsilon(\mathbf{k})+\frac{W_{0}}{2}\right)+1}\right\}
\end{aligned}
$$

By plugging the ansatz $\mathbf{W}=W_{0} \mathbf{n}_{z}+\chi$, with $\chi$ orthogonal to $\mathbf{n}_{z}$, into the $\sigma$-model action (4.21), one easily sees that the coefficient $C_{\nabla}$ just calculated coincides with the corresponding coefficient in the $\sigma$-model action.

For the Hubbard model, we can deduce sufficient conditions for the linear stability of the ferromagnetic mean-field configuration (i.e., for $C_{\nabla}$ to be negative) from (4.40), for large values of $\beta W_{0}$. To get a rough idea of a sufficient condition for $C_{\nabla}$ to be negative, we approximate the Fermi surfaces of the spin-up and spin-down bands by spheres. We denote by $v_{F}^{ \pm}$the Fermi velocities and by $k_{F}^{ \pm}$the Fermi momenta of the spin-up and spin-down bands, respectively, with

$$
\left|\nabla \epsilon\left(k_{F}^{ \pm}\right)\right|=v_{F}^{ \pm},
$$

by $A_{F}^{ \pm}$the areas of the Fermi surfaces of the spin-up and spin-down bands, respectively, and by $V_{F}^{ \pm}$the respective volumes. Then, for large values of $\beta W_{0}$, a sufficient condition for linear stability can be read off from (4.40):

$$
\frac{W_{0}}{2}\left(A_{F}^{+} v_{F}^{+}+A_{F}^{-} v_{F}^{-}\right)>v_{\max }^{2}\left(V_{F}^{-}-V_{F}^{+}\right),
$$

where $v_{\max }=\max \left\{|\nabla \epsilon(\mathbf{k})| \mid \epsilon(\mathbf{k})-W_{0} / 2<0<\epsilon(\mathbf{k})+W_{0} / 2\right\}$. For weak fillings

$$
\epsilon(\mathbf{k}) \approx \delta|\mathbf{k}|^{2}-\mu
$$

is a good approximation of $\epsilon$ for wave vectors, $\mathbf{k}$, corresponding to occupied states (with spin down). The stability condition (4.41) can then be expressed in terms of the parameters $W_{0}, \mu$ and $\delta$. The parameters $W_{0}$ and $\delta$ are supposed to be positive, 
whereas $\mu$ can take negative values, too. From the equations

$$
\epsilon\left(k_{F}^{ \pm}\right) \pm \frac{W_{0}}{2}=0
$$

and (4.42) we get

$$
\begin{aligned}
v_{\max } & =v_{F}^{-}, \\
v_{F}^{ \pm} & =2 \delta k_{F}^{ \pm}=2 \sqrt{\delta\left(\mu \mp W_{0} / 2\right)}, \\
A_{F}^{ \pm} & =4 \pi\left(k_{F}^{ \pm}\right)^{2}, \\
V_{F}^{ \pm} & =\frac{4 \pi}{3}\left(k_{F}^{ \pm}\right)^{3} .
\end{aligned}
$$

We thus arrive at the following conditions for linear stability of ferromagnetic mean-field configurations $\left(C_{\nabla}<0\right)$.

The existence of a Fermi surface, Eq. (4.43), yields the bound

$$
W_{0} \leq c_{1} \delta-\mu,
$$

where $c_{1}$ is a positive numerical constant of $\mathcal{O}(1)$. Next, a sufficient condition for inequality (4.41) to hold, is given by

$$
W_{0} \geq c_{2} \mu,
$$

for a positive numerical constant $c_{2}$ of $\mathcal{O}(1)$. From (4.44) and (4.45) we get the condition for the band:

$$
\mu \leq c_{3} \delta,
$$

where $c_{3}$ is a numerical constant of $\mathcal{O}(1)$.

In the regime defined by (4.44) and (4.45), the dimensionless coefficient $\beta C_{\nabla}$ is of the order

$$
\left|\beta C_{\nabla}\right| \approx \beta A_{F}^{-} v_{F}^{-} \approx \beta \delta^{-1 / 2} W_{0}^{3 / 2}=\beta W_{0}\left(W_{0} / \delta\right)^{1 / 2},
$$

from which we see that a flat band and large values of the exchange field $W_{0}$ favour stability of the ferromagnetic state.

The mean-field Eq. (4.7) for the one-band Hubbard model,

$$
W_{0}=\frac{2 U_{0}}{3} \frac{V_{F}^{-}-V_{F}^{+}}{(2 \pi)^{3}},
$$

and inequalities (4.44) and (4.45) can be simultaneously fulfilled if the on-site repulsion $U_{0}$ is chosen appropriately (depending on $\mu$ and $\delta$ ). Hence, at low temperatures, for small $\delta$ and an appropriate choice of $\mu$ and $U_{0}$, the less than half-filled one-band Hubbard model has quadratically stable ferromagnetic meanfield solutions.

Details of these and other related calculations will appear in Ref. 18. 


\subsection{Magnon Dynamics and the $W Z$-Term}

In this subsection we consider the Wess-Zumino-term in (4.21). We first consider an $x$-independent exchange field $\mathbf{W}(t)$ of constant length $|\mathbf{W}(t)|=W_{0}$ and calculate the variation of the first term on the R.S. of (3.40) for $\epsilon=0$, given a variation, $\delta \mathbf{W}$, of the exchange field:

$$
\delta \ln \operatorname{det}\left(D_{0, \mathbf{w}}\right)=\operatorname{tr}\left(\delta D_{0, \mathbf{w}}\left(D_{0, \mathbf{w}}^{*} D_{0, \mathbf{w}}\right)^{-1} D_{0, \mathbf{w}}^{*}\right),
$$

where

$$
\delta D_{0, \mathbf{W}}=\frac{1}{2} \delta \mathbf{W}(t) \cdot \boldsymbol{\sigma} \equiv \delta W(\mathrm{t}) .
$$

In order to evaluate the R.S. of (4.48), we expand $\left(D_{0, \mathbf{w}}^{*} D_{0, \mathbf{w}}\right)^{-1}$ in a Neumann series in powers of $\left(\partial_{t} W\right)$,

$$
\left(D_{0, \mathbf{W}}^{*} D_{0, \mathbf{W}}\right)^{-1}=\Lambda^{-1}+\Lambda^{-1}\left(\partial_{t} W\right) \Lambda^{-1}+\ldots,
$$

where $\Lambda=-\partial_{t}^{2}+(1 / 4) W_{0}^{2}$. Plugging this series into (4.48), we find that

$$
\delta \ln \operatorname{det}\left(D_{0, \mathbf{W}}\right)=\delta S^{(1)}(\mathbf{W})+\delta S^{(2)}(\mathbf{W})+\ldots .
$$

where

$$
\begin{aligned}
& \delta S^{(1)}(\mathbf{W})=\operatorname{tr}\left(\delta W \Lambda^{-1} D_{0, \mathbf{W}}^{*}\right), \\
& \delta S^{(2)}(\mathbf{W})=\operatorname{tr}\left(\delta W \Lambda^{-1}\left(\partial_{t} W\right) \Lambda^{-1} D_{0, \mathbf{W}}^{*}\right),
\end{aligned}
$$

etc.. Using the cyclicity of the trace and the fact that $\operatorname{Tr}(\sigma)=0$ (where " $\operatorname{tr}$ " is the trace on $2 \times 2$ matrices), we see that

$$
\delta S^{(1)}(\mathbf{W})=\alpha\left(W_{0}\right) \int_{0}^{\beta} d t \operatorname{Tr}(W(t) \delta W(t))
$$

where

$$
\alpha\left(W_{0}\right)=\beta^{-1} \sum_{k_{0} \in Z_{\beta}} \frac{1}{k_{0}^{2}+(1 / 4) W_{0}^{2}},
$$

and $Z_{\beta}=(\pi / \beta)(2 \mathbb{Z}+1)$. Since

$$
\operatorname{Tr}(W(t) \delta W(t))=\frac{1}{2} \delta \operatorname{Tr}\left(W(t)^{2}\right),
$$

$\delta S^{(1)}$ only depends on variations of the length of $\mathbf{W}(t)$ and vanishes if the length is held fixed. By (4.51),

$$
\delta S^{(2)}(\mathbf{W})=\delta S_{I}^{(2)}(\mathbf{W})+\delta S_{I I}^{(2)}(\mathbf{W}),
$$


where

$$
\delta S_{I}^{(2)}(\mathbf{W})=-\operatorname{tr}\left(\delta W \Lambda^{-1}\left(\partial_{t} W\right) \Lambda^{-1} \partial_{t}\right)=-\frac{1}{2} \sum_{j=1}^{3} \operatorname{tr}\left(\delta W_{j} \Lambda^{-1}\left(\partial_{t} W\right)_{j} \Lambda^{-1} \partial_{t}\right)
$$

and

$$
\delta S_{I I}^{(2)}(\mathbf{W})=\operatorname{tr}\left(\delta W \Lambda^{-1}\left(\partial_{t} W\right) \Lambda^{-1} W\right)=-\frac{i}{4} \sum_{j, l, m} \epsilon_{j l m} \operatorname{tr}\left(\delta W_{j} \Lambda^{-1}\left(\partial_{t} W\right)_{l} \Lambda^{-1} W_{m}\right)
$$

Here we have used that $\operatorname{tr}\left(\sigma_{j} \sigma_{l}\right)=2 \delta_{j l}$ and $\operatorname{tr}\left(\sigma_{j} \sigma_{l} \sigma_{m}\right)=-2 i \epsilon_{j l m}$. Since $\partial_{t}, \Lambda$ and $W_{j}(t)$ are real operators, $\delta S_{I}^{(2)}$ is real and $\delta S_{I I}^{(2)}$ purely imaginary. The real part has been calculated in the last subsection. Turning to $\delta S_{I I}^{(2)}(\mathbf{W})$, we observe that

$$
\begin{aligned}
\delta S_{I I}^{(2)}(\mathbf{W}) & =\operatorname{tr}\left(W \delta W\left(\partial_{t} W\right) \Lambda^{-2}\right)+\text { higher derivative terms } \\
& =-\frac{i}{4} \gamma\left(W_{0}\right) \int_{0}^{\beta} d t \sum_{j, l, m} \epsilon_{j l m} W_{j}(t) \delta W_{l}(t) \partial_{t} W_{m}(t)+\text { h.d.t. } \\
& =-\frac{i}{2} \int_{0}^{\beta} d t \hat{\mathbf{W}}(t) \cdot\left(\delta \hat{\mathbf{W}}(t) \wedge \partial_{t} \hat{\mathbf{W}}(t)\right)+\mathcal{O}\left(\left(\beta W_{0}\right)^{-1}\right)+\text { h.d.t. },
\end{aligned}
$$

where, in the last equation, we have used that

$$
\gamma\left(W_{0}\right):=\beta^{-1} \sum_{k_{0} \in Z_{\beta}} \frac{1}{\left(k_{0}^{2}+(1 / 4) W_{0}^{2}\right)^{2}} \underset{\beta W_{0} \rightarrow \infty}{\longrightarrow} \frac{2}{W_{0}^{3}} .
$$

We note that h.d.t. $\sim \mathcal{O}\left(\left(\beta W_{0}\right)^{-1}\right)$.

Recall that $\mathbf{W}(t)$ has periodic boundary conditions at $t=0, \beta$. Viewing the imaginary-time circle $[0, \beta)$ as the equator of a sphere $S_{\beta}^{2}$ of radius $\beta /(2 \pi)$, we may extend the unit-vector field $\hat{\mathbf{W}}(t)$ from the equator of $S_{\beta}^{2}$ to a continuous unit-vector field $\hat{\mathbf{W}}(t, s), 0 \leq s \leq \beta / 2$, on the entire sphere in an arbitrary way, but with $\hat{\mathbf{W}}(t, \beta / 4)=\hat{\mathbf{W}}(t)$. The first term on the R.S. of (4.52) then turns out to be the variation of the functional

$$
\begin{aligned}
S_{W Z}(\mathbf{W}) & =-\frac{i}{2} \int_{0}^{\beta} d t \int_{0}^{\beta / 4} d s \hat{\mathbf{W}}(t, s) \cdot\left(\partial_{s} \hat{\mathbf{W}}(t, s) \wedge \partial_{t} \hat{\mathbf{W}}(t, s)\right) \\
& =-\frac{i}{2} \int_{S^{2, N}} \hat{\mathbf{W}} \cdot(d \hat{\mathbf{W}} \wedge d \hat{\mathbf{W}}),
\end{aligned}
$$

where " $d$ " denotes the exterior derivative, and $S^{2, N}$ is the northern hemisphere of $S^{2}$. We recognize (4.54) to be the Wess-Zumino term in (4.21) for an $x$-independent 
(i.e., ferromagnetically ordered) exchange field. The corresponding coefficient $C_{W Z}$ depends on $\epsilon$ and will be determined later on. The R.S. of (4.54) is expressed in standard differential-form notation, which makes it manifest that $S_{W Z}(\mathbf{W})$ is independent of the radius, $\beta / 2 \pi$, of the sphere.

The leading term on the R.S. of (4.52) is also the variation of

$$
S_{W Z}^{\prime}(\mathbf{W})=\frac{i}{2} \int_{S^{2, S}} \hat{\mathbf{W}} \cdot(d \hat{\mathbf{W}} \wedge d \hat{\mathbf{W}}),
$$

where $S^{2, S}$ is the southern hemisphere of $S^{2}$. One observes that

$$
S_{W Z}(\mathbf{W})-S_{W Z}^{\prime}(\mathbf{W})=-\frac{i}{2} \int_{S^{2}} \hat{\mathbf{W}} \cdot(d \hat{\mathbf{W}} \wedge d \hat{\mathbf{W}})=2 \pi i n,
$$

where $n=n(\hat{\mathbf{W}}) \in \mathbb{Z}$ is the degree of the map $\hat{\mathbf{W}}: S^{2} \rightarrow S^{2}$. It follows that, apparently, the Wess-Zumino action $S_{W Z}$ is only determined modulo an integer multiple of $2 \pi i$. Due to (4.55), $\exp \left(S_{W Z}(\mathbf{W})\right)$ is a single-valued functional of $\mathbf{W}$. field

If the exchange field $\mathbf{W}(x, t)$ is antiferromagnetically ordered, i.e., the Néel

$$
\mathbf{N}(x, t) \equiv(-1)^{|x|} \mathbf{W}(x, t)=W_{0} \mathbf{n}
$$

is a constant field pointing in the direction of some unit vector $\mathbf{n}$, the Wess-Zumino term in (4.21) tends to 0 in the scaling limit. It follows that, in antiferromagnetically ordered states, at sufficiently low temperatures, there are gapless Goldstone bosons with dispersion

$$
\omega(\mathbf{k}) \approx v|\mathbf{k}|, \quad(|\mathbf{k}| \approx 0)
$$

for some velocity $v$.

In ferromagnetically ordered states, however, the Wess-Zumino term survives. Using (4.37) and (4.54), the coefficient $C_{W Z}$ in (4.21) is found to be

$$
C_{W Z}=-\frac{i}{2} \frac{\left(V_{F}^{-}-V_{F}^{+}\right)}{(2 \pi)^{3}},
$$

in the limit where $\beta W_{0} \rightarrow \infty$. Had we normalized the Fourier modes and were we in a finite volume, we would find that $\exp \left(\tilde{S}_{\text {eff }}(\mathbf{W})\right)$ is single-valued. From (4.21) we derive the equation of motion

$$
C_{W Z} \hat{\mathbf{W}}_{x} \wedge \dot{\mathbf{W}}_{x}=\sum_{y} \tilde{K}(x-y) \hat{\mathbf{W}}_{y}+\alpha \hat{\mathbf{W}}_{x},
$$

with $\quad \hat{\mathbf{W}}_{x}(t)=\hat{\mathbf{W}}(x, t), \quad \dot{\hat{\mathbf{W}}}_{x}=\partial_{t} \hat{\mathbf{W}}_{x}, \quad$ where $\quad \tilde{K}(x-y)=W_{0}^{2} K(x-y)+$ $C_{\nabla} \delta_{|x-y|, 1}$, and where $\alpha$ is a Lagrange multiplier arising from the constraint $\left|\hat{\mathbf{W}}_{x}(t)\right|^{2}=1$, for all $x$ and all $t$. Taking the vector product of (4.58) with $\hat{\mathbf{W}}_{x}$, and 
doing the Wick rotation from imaginary time back to real time, ${ }^{(28)}$ we obtain the equation of motion for magnons in a ferromagnet

$$
i C_{W Z} \dot{\hat{\mathbf{W}}}_{x}=\hat{\mathbf{W}}_{x} \wedge \sum_{y} \tilde{K}(x-y) \hat{\mathbf{W}}_{y},
$$

which is the well known Landau-Lifshitz equation; see also Ref. 19.

If $J$, and hence $K$, are of short range and if the equilibrium state of the system were ferromagnetically ordered, i.e.,

$$
\mathbf{W}(x, t)=W_{0} \mathbf{n}+\chi(x, t), \quad W_{0}>0,
$$

where $\mathbf{n}$ is a unit vector and $\chi(x, t)$ is assumed to be small, then the dispersion of magnons is found to be

$$
\omega(\mathbf{k})=\frac{|\mathbf{k}|^{2}}{2 M}, \quad \text { for some constant } M>0,
$$

as expected. If $\mathbf{n}$ points in the $z$-direction then $\chi$ lies essentially in the $x-y$ plane. We define

$$
\phi(x, t)=\chi_{1}(x, t)+i \chi_{2}(x, t) .
$$

Passing to the formal continuum limit, the action functional for the complex magnon field $\phi$ of a ferromagnet is seen to be given by

$$
S_{\text {magnon }}(\bar{\phi}, \phi)=\int_{0}^{\beta} d t \int d x\left[\bar{\phi}(x, t) \partial_{t} \phi(x, t)+\frac{1}{2 M} \bar{\phi}(x, t)(\Delta \phi)(x, t)\right] \text {, }
$$

which is the action for a system of conserved non-relativistic bosons with vanishing chemical potential. Since $W_{1}=\chi_{1}=(\phi+\bar{\phi}) / 2$ is an observable field, the chemical potential actually necessarily vanishes, and there is no "magnon condensation"; (just like photons cannot form any Bose condensates).

\subsection{Absence of Symmetry Breaking in One and Two Dimensions at Positive Temperatures}

For $\beta<\infty$, we can study the fluctuations of the modes $\mathbf{W}\left(k_{0}, \mathbf{k}\right)$ of the exchange field with $k_{0}=0$, i.e., of $\int_{0}^{\beta} \mathbf{W}(x, t) d t$.

We set

$$
\int_{0}^{\beta} \mathbf{W}(x, t) d t=\beta\left(\mathbf{W}^{m f}+\chi_{l}(x) \mathbf{n}_{z}+\chi_{t}(x)\right)
$$


where $\mathbf{W}^{m f}$ is a solution of the ferromagnetic or the antiferromagnetic mean-field equation. Then, in $d$ spatial dimensions, we conclude from our stability analysis

$$
\left\langle\chi_{t}(x)^{2}\right\rangle_{\beta, \mu} \propto \int_{B_{\Gamma}} \frac{d^{d} k}{k^{2}}
$$

which, for $d=1$ and 2, is infrared-divergent. Thus, transversal fluctuations around the mean field are gigantic and hence destroy long-range order; (this is the MerminWagner theorem, see, e.g., Ref. 6).

In two dimensions, there is an alternative way to understand the absence of ordering when $\beta<\infty$ : Setting $\mathbf{W}(x, t)=\tilde{\mathbf{W}}(x)$ (ferromagnetic short-range order), or $\mathbf{W}(x, t)=(-1)^{|x|} \tilde{\mathbf{W}}(x)$ (antiferromagnetic short-range order), where $\tilde{\mathbf{W}}(x)$ is slowly varying, and passing to the formal continuum limit (lattice spacing $\rightarrow 0$ ), one finds that the action $S_{\text {eff }}(\mathbf{W})$ has (approximate) critical points, indeed local maxima, on time-independent configurations $\tilde{\mathbf{W}}(x)$ with the properties:

$$
|\tilde{\mathbf{W}}(x)| \approx W_{0}, \text { for all } x,
$$

where $W_{0}$ is a solution of the (ferromagnetic or antiferromagnetic) mean-field equation,

$$
\tilde{\mathbf{W}}(x) \rightarrow W_{0} \mathbf{n}, \quad \text { as } \quad|x| \rightarrow \infty,
$$

where $\mathbf{n}$ is an arbitrary unit vector, and the degree of the map $\tilde{\mathbf{W}}(x)$, as measured by the integer ("winding number")

$$
\frac{1}{4 \pi W_{0}^{3}} \int d^{2} x \tilde{\mathbf{W}}(x) \cdot\left(\frac{\partial \tilde{\mathbf{W}}(x)}{\partial x_{1}} \wedge \frac{\partial \tilde{\mathbf{W}}(x)}{\partial x_{2}}\right),
$$

is non-zero. Such configurations are called "instantons." The contributions of instanton configurations to a functional integral, such as (3.43), destroy longrange order.

Remark: Generally speaking, it is hard to justify the use of steepest descent in approximately evaluating functional integrals, such as (3.41) and (3.43), because there isn't any large constant $\mathcal{N}=h^{-1}$ multiplying $S_{\text {eff }}(\mathbf{W})$. If, however, we place $\mathcal{N}$ identical species of spin- $\frac{1}{2}$-fermions on each site $x \in \Gamma$, coupled to each other only through exchange interactions between the total spin operators, and if we set $K=\mathcal{N} K_{0}$ then $S_{\text {eff }}(\mathbf{W}) \equiv S_{\text {eff }}^{\left(K_{0}\right)}(\mathbf{W})$ is replaced by $\mathcal{N} S_{\text {eff }}^{\left(K_{0}\right)}(\mathbf{W})$, and steepest descent becomes reliable.

\section{REFLECTION POSITIVITY AND PHASE TRANSITIONS IN HEISENBERG-MODELS}

In order to establish the existence of phase transitions in some of the models introduced in Sec. 3, we recall the method of reflection positivity ${ }^{(4,5,20,21)}$. For 
simplicity, we choose the lattice $\Gamma$ to be given by $\mathbb{Z}^{d}$. We decompose $\Gamma$ into two equal halves

$$
\Gamma_{+}=\left\{x \in \Gamma \mid x_{1}>0\right\}, \quad \Gamma_{-}=\left\{x \in \Gamma \mid x_{1} \leq 0\right\},
$$

where $x_{j}$ is the $j^{\text {th }}$ component of $x \in \Gamma$. Let $\Pi$ be the plane in $\mathbb{R}^{d}$ orthogonal to the 1-direction at height $x_{1}=\frac{1}{2}$, and let $\vartheta$ denote reflection at $\Pi$. Clearly, $\vartheta$ maps $\Gamma_{-}$onto $\Gamma_{+}$, and conversely.

An exchange coupling matrix, $J(x, y)$, is said to be reflection positive iff

$$
\sum_{x, y} \overline{f(x)} J(x, \vartheta y) f(y) \geq 0,
$$

for all functions $f$ on $\Gamma$ with supp $f \subseteq \Gamma_{+}$. Note that this condition is independent of the diagonal elements, $J(x, x)$, of $J$; so, by choosing them appropriately, we can always ensure that $J$ is either positive-definite or negative-definite. By computing some integrals with a Gaussian measure of mean 0 and covariance $-J^{-1}$ ( $J$ negative-definite) we see that if $J$ is reflection positive then so is $-J^{-1}$; see e.g. Refs. 20,21. Furthermore, if $J$ is reflection-positive then so is $-\widetilde{J}$, where $\widetilde{J}$ is given by

$$
\widetilde{J}(x, y)=(-1)^{|x+y|} J(x, y)=(-1)^{|x|+|y|} J(x, y)
$$

with $|x|=\sum_{j=1}^{d} x_{j}$. (Note that if $J$ is positive-definite then $\widetilde{J}$ is positive-definite, too.) The following simple calculation proves our claim. Let

$$
\tilde{f}(x)=(-1)^{|x|} f(x), \quad \operatorname{supp} f \subseteq \Gamma_{+} .
$$

Since $J$ is assumed to be reflection-positive,

$$
\begin{aligned}
0 & \leq \sum_{x, y} \overline{\tilde{f}(x)} J(x, \vartheta y) \tilde{f}(y) \\
& =\sum_{x, y} \overline{f(x)}(-1)^{|x|+|y|} J(x, \vartheta y) f(y) \\
& =-\sum_{x, y} \overline{f(x)}(-1)^{|x+\vartheta y|} J(x, \vartheta y) f(y) \\
& =-\sum_{x, y} \overline{f(x)} \widetilde{J}(x, \vartheta y) f(y),
\end{aligned}
$$

for an arbitrary function $f$ with supp $f \subseteq \Gamma_{+}$(hence supp $\tilde{f} \subseteq \Gamma_{+}$). These considerations are summarized as follows:

4.14If $J$ is reflection-positive then $-K:=-J^{-1}$ is reflection-positive, too; 
and

$$
\begin{gathered}
\text { if }-J \text { is reflection-positive (i.e., } J \text { is reflection-negative) } \\
\text { then }-\widetilde{K}:=-\widetilde{J^{-1}} \text { is reflection-positive, too. }
\end{gathered}
$$

In both situations, we can choose the diagonal elements of $J$ such that $J$ and $K$ are positive-, or negative-definite.

A reflection-positive matrix $J$ is called ferromagnetic, while a reflectionnegative matrix is called antiferromagnetic. It is easy to see that, for nearestneighbour exchange couplings, these notions of "ferro-" and "antiferromagnetic" coincide with the familiar ones.

Let $J$ be ferromagnetic and negative-definite. Then the Gaussian measure

$$
d \mu_{-J}(\vec{\phi})=\text { const } \cdot e^{\frac{1}{2} \sum_{x, y} \vec{\phi}(x) J(x, y) \vec{\phi}(y)} \prod_{x} d^{n} \phi(x),
$$

where $\vec{\phi}(x) \in \mathbb{R}^{n}, n=1,2,3, \ldots$, and the constant is chosen such that $d \mu_{-J}$ is normalized, is reflection-positive, in the sense that

$$
\int d \mu_{-J}(\phi) \overline{(\Theta F)(\phi)} F(\phi) \geq 0,
$$

for an arbitrary bounded function $F$ that depends only on the variables $\{\vec{\phi}(x) \mid x \in$ $\left.\Gamma_{+}\right\}$; the space of all such functions is denoted by $\mathcal{F}_{+}$. The function $\Theta F$ in (5.6) is defined by

$$
(\Theta F)(\phi)=F\left(\phi_{\vartheta}\right),
$$

where $\phi_{\vartheta}(x)=\phi(\vartheta x)$. If $\phi^{(1)}, \ldots, \phi^{(l)}$ are independent Gaussian random fields with distributions given by $d \mu_{-J^{(t)}}\left(\phi^{(t)}\right)$, where $J^{(t)}$ is ferromagnetic and $J^{(t)}<$ $0, t=1, \ldots, l$, then the distribution

$$
d \mu(\phi)=\prod_{t=1}^{l} d \mu_{-\tau^{(t)} J^{(t)}}\left(\phi^{(t)}\right)
$$

of the random field $\phi$ given by

$$
\phi(x, t)=\phi^{(t)}(x), t=1, \ldots, l,
$$

is reflection-positive, for arbitrary $\tau^{(t)}>0, t=1, \ldots, l$; as is easily seen.

These considerations have the following consequence that will play a crucial role in our analysis of phase transitions: Let $\mathbf{W}(x, t), x \in \Gamma, t \in[0, \beta)$, with $\mathbf{W}(x, t+\beta)=\mathbf{W}(x, t)$, be the imaginary time exchange field, and let $d \mu_{K}(\mathbf{W})=\exp \left(S_{K}(\mathbf{W})\right) \mathcal{D} \mathbf{W}$ be its Gaussian distribution, as introduced in Sec. 3, (3.27) through (3.31). If the exchange couplings $J$ are ferromagnetic (in the sense 
specified above) and positive-definite then $-K=-J^{-1}$ is ferromagnetic and negative-definite; hence the Gaussian measure

$$
d \mu_{K}(\mathbf{W}) \text { is reflection-positive, }
$$

as follows from (3.29), (5.6) and (5.8).

Next, we suppose that the exchange couplings $J$ are antiferromagnetic (in the sense specified above). Then, by $(5.5),-\widetilde{K}=-\widetilde{J^{-1}}$ is reflection-positive and negative-definite, for $J$ positive-definite. We introduce a random field, N, ("N" for "Néel"), by setting

$$
\mathbf{N}(x, t)=(-1)^{|x|} \mathbf{W}(x, t) .
$$

If $\mathbf{W}$ has distribution $d \mu_{K}(\mathbf{W})$, with $K=J^{-1}$, then $\mathbf{N}$ has distribution $d \mu_{\widetilde{K}}(\mathbf{N})$, and hence

$$
d \mu_{\widetilde{K}}(\mathbf{N}) \text { is reflection-positive. }
$$

We set

$$
\mathbf{N}_{\vartheta}(x, t)=\mathbf{N}(\vartheta x, t)
$$

and (see (5.7))

$$
(\Theta F)(\mathbf{N})=F\left(\mathbf{N}_{\vartheta}\right),
$$

for an arbitrary function $F$ of $\{\mathbf{N}(x, t) \mid x \in \Gamma, t \in[0, \beta)\}$.

We consider the models introduced in Sec. 3 without hopping term but with $\mathcal{N}$ Fermion species. They have an action functional given by

$$
\begin{aligned}
\bar{S}(\bar{C}, C ; \mathbf{W})= & \sum_{a=1}^{\mathcal{N}} \int_{0}^{\beta} d t \sum_{x}\left[\bar{C}_{s}^{(a)}(x, t)\left(\frac{\partial}{\partial t}-\mu\right) C_{s}^{(a)}(x, t) v_{a} \mathbf{W}(x, t)\right. \\
& \left.\times \mathbf{S}^{(a)}(x, t)\right]-\frac{1}{2} \int_{0}^{\beta} d t\left(\sum_{x, y} \mathbf{W}(x, t) K(x-y) \mathbf{W}(y, t)\right),
\end{aligned}
$$

where $v_{a}= \pm 1$, for all $a=1, \ldots, \mathcal{N}$, and $\hat{K}(k)=\left(\frac{4}{3} U_{0}+\hat{J}_{\neq}(k)\right)^{-1}$; see (3.20), (3.29), and (3.32) through (3.34). Since the first term on the R.S. of (5.14) does not couple different sites in the lattice, the effective action of the exchange field $\mathbf{W}$, see (3.36) and (3.40), is given by

$$
S_{\mathrm{eff}}(\mathbf{W})=-\frac{1}{2} \int_{0}^{\beta} d t\left(\sum_{x, y} \mathbf{W}(x, t) K(x-y) \mathbf{W}(y, t)\right)
$$




$$
+\sum_{a} \sum_{x} \ln \operatorname{det}\left(D_{v_{a}} \mathbf{w}(x, \cdot)\right)
$$

with $D_{\mathbf{W}}$ given in (3.38), for $\hat{t}(x) \equiv 0$. We define

$$
\begin{aligned}
f_{x}(\mathbf{N}) & =\prod_{a=1}^{\mathcal{N}} \operatorname{det}\left(D_{v_{a}(-1)^{|x|} \mathbf{N}(x, \cdot)}\right) \\
& =\prod_{a=1}^{\mathcal{N}} \operatorname{det}\left(D_{v_{a} \mathbf{w}(x, \cdot)}\right),
\end{aligned}
$$

Then it follows that

$$
\begin{aligned}
\overline{\Theta f_{x}(\mathbf{N})} & =\overline{f_{x}\left(\mathbf{N}_{\vartheta}(x, \cdot)\right)} \\
& =\prod_{a=1}^{\mathcal{N}} \overline{\operatorname{det}\left(D_{v_{a}(-1)^{|x|} \mathbf{N}_{\vartheta}(x, \cdot)}\right)} \\
& =\prod_{a=1}^{\mathcal{N}} \overline{\operatorname{det}\left(D_{-v_{a}(-1)^{|\vartheta x|} \mathbf{N}(\vartheta x, \cdot)}\right)} \\
& =\prod_{a=1}^{\mathcal{N}} \operatorname{det}\left(D_{v_{a}(-1)^{|\vartheta x|} \mathbf{N}(\vartheta x, \cdot)}\right) \\
& =f_{\vartheta x}(\mathbf{N}),
\end{aligned}
$$

where, in the third equation, we have used that $(-1)^{|x|}=-(-1)^{|\vartheta x|}$, and, in the fourth equation, we have inserted identity (4.27). Equation (5.17) implies that

$$
\overline{\Theta\left(\prod_{x \in \Gamma_{+}} f_{x}(\mathbf{N})\right)}=\prod_{x \in \Gamma_{-}} f_{x}(\mathbf{N}) .
$$

From this equation and Eqs. (5.11) and (5.6) we conclude the following simple, but quite fundamental

Result A Suppose the exchange couplings $J$ are antiferromagnetic (in the sense introduced above) and positive-definite. We set

$$
\begin{aligned}
d \mu_{\mathrm{eff}}(\mathbf{N}) & =\text { const } \cdot \prod_{x} f_{x}(\mathbf{N}) d \mu_{\widetilde{K}}(\mathbf{N}) \\
& =\text { const }^{\prime} \cdot \exp \left(S_{\text {eff }}(\mathbf{N})\right) \mathcal{D} \mathbf{N},
\end{aligned}
$$

where the constants are chosen such that $\int d \mu_{\text {eff }}(\mathbf{N})=1$, and

$$
S_{\mathrm{eff}}(\mathbf{N}):=-\frac{1}{2} \int_{0}^{b} d t\left(\sum_{x, y} \mathbf{N}(x, t) \widetilde{K}(x-y) \mathbf{N}(y, t)\right)
$$




$$
+\sum_{a} \sum_{x} \ln \operatorname{det}\left(D_{v_{a}(-1)^{|x|} \mathbf{N}(x, \cdot)}\right)
$$

with $\widetilde{K}=\widetilde{J^{-1}}$; see (5.10), (5.11), (5.15).

Then the measure $d \mu_{\mathrm{eff}}(\mathbf{N})$ is reflection-positive, in the sense that

$$
\int d \mu_{\mathrm{eff}}(\mathbf{N}) \overline{(\Theta F)(\mathbf{N})} F(\mathbf{N}) \geq 0,
$$

for arbitrary functions $F \in \mathcal{F}_{+}$(where $\mathcal{F}_{+}$is defined right above (5.7)).

Next, we suppose that the number of fermion species, $\mathcal{N}=2 \mathcal{M}$ is even, with

$$
v_{1}=\ldots=v_{\mathcal{M}}=1, \quad v_{\mathcal{M}+1}=\ldots=v_{2 \mathcal{M}}=-1 .
$$

We define

$$
g_{x}(\mathbf{W})=\operatorname{det}\left(D_{\mathbf{W}(x, \cdot)}\right)^{\mathcal{M}} \operatorname{det}\left(D_{-\mathbf{W}(x, \cdot)}\right)^{\mathcal{M}} .
$$

Identity (4.27) then implies that $g_{x}(\mathbf{W}) \geq 0$, for all $x \in \Gamma$, and, setting

$$
\mathbf{W}_{\vartheta}(x, t)=\mathbf{W}(\vartheta x, t),
$$

we find that

$$
\overline{\Theta g_{x}(\mathbf{W})}=\Theta g_{x}(\mathbf{W})=g_{\vartheta x}(\mathbf{W}) .
$$

Defining

$$
\begin{aligned}
d \mu_{\mathrm{eff}}(\mathbf{W}) & =\text { const } \cdot \prod_{x} g_{x}(\mathbf{W}) d \mu_{K}(\mathbf{W}) \\
& =\text { const }^{\prime} \cdot \exp \left(S_{\text {eff }}(\mathbf{W})\right) \mathcal{D} \mathbf{W},
\end{aligned}
$$

where $K=J^{-1}, S_{\mathrm{eff}}(\mathbf{W})$ is as in (5.15), and the constants are chosen such that $\int d \mu_{\mathrm{eff}}(\mathbf{W})=1$, we arrive at

Result F Suppose the exchange couplings $J$ are ferromagnetic (in the sense introduced above) and assumption (5.23) holds. Then the measure $d \mu_{\text {eff }}(\mathbf{W})$ is reflection-positive, i.e.

$$
\int d \mu_{\mathrm{eff}}(\mathbf{W}) \overline{(\Theta F)(\mathbf{W})} F(\mathbf{W}) \geq 0,
$$

for arbitrary functions $F \in \mathcal{F}_{+}$.

From Results A and F one obtains the following infrared (spin-wave) bounds, originally discovered in Ref. 5 and generalized in Refs. 20, 21. 
(IRA) Under the hypotheses of Result A (in particular, $J$ antiferromagnetic), one has the inequalities

$$
\begin{aligned}
0 & \leq\left\langle\widehat{\mathbf{N}}\left(\mathbf{k}, k_{0}\right) \cdot \widehat{\mathbf{N}}\left(-\mathbf{k},-k_{0}\right)\right\rangle_{\beta, \mu} \\
& \leq W_{0}^{2} \delta_{0}(\mathbf{k}) \delta_{k_{0}, 0}+\beta^{-1}(\widehat{\widetilde{K}}(\mathbf{k})-\widehat{\widetilde{K}}(0))^{-1}
\end{aligned}
$$

for some constant $W_{0}^{2} \geq 0$. Note that $\widehat{\mathbf{N}}\left(-\mathbf{k},-k_{0}\right)=\widehat{\mathbf{N}}\left(\mathbf{k}, k_{0}\right)$, because $\mathbf{N}(x, t)$ is real.

If $W_{0}^{2}$ is strictly positive then the state $\langle(\cdot)\rangle_{\beta, \mu}$ exhibits long-range order, and if we couple $\mathbf{N}$ to an arbitrarily small external field, $\varepsilon \mathbf{n},|\mathbf{n}|=1$, then

$$
\lim _{\varepsilon \rightarrow 0}\langle\mathbf{N}(x, t)\rangle_{\beta, \mu, \varepsilon \mathbf{n}}=W_{0} \mathbf{n} .^{7}
$$

Inequality (5.28) implies that, for an arbitrary bounded function $f(x, t)$ of rapid decay in $x \in \Gamma$ and periodic in $t \in[0, \beta)$,

$$
\begin{aligned}
0 & \leq\left\langle|\mathbf{N}(f)|^{2}\right\rangle_{\beta, \mu} \\
& \leq \beta^{2} W_{0}^{2}|\hat{f}(0,0)|^{2}+\beta \sum_{k_{0} \in \frac{2 \pi}{\beta} \mathbb{Z}} \int_{B_{\Gamma}} d \mathbf{k} \frac{\left|\hat{f}\left(\mathbf{k}, k_{0}\right)\right|^{2}}{\widehat{\widehat{K}}(\mathbf{k})-\widehat{\widehat{K}}(0)},
\end{aligned}
$$

where $\mathbf{N}(f)=\int_{0}^{\beta} d t \sum_{x} \mathbf{N}(x, t) f(x, t)$.

Returning to identities (3.42) and (3.43), with

$$
\mathbf{S}(x, t)=\sum_{a=1}^{\mathcal{N}} v_{a} \mathbf{S}^{(a)}(x, t),
$$

and recalling that $\mathbf{W}(x, t)=(-1)^{|x|} \mathbf{N}(x, t)$, we conclude from (5.30) that

$$
\begin{aligned}
0 & \leq\left\langle\left(\int_{0}^{\beta} \mathbf{S}(0, t) d t\right)^{2}\right\rangle_{\beta, \mu} \\
& \leq-\beta \widetilde{K}(0)+\beta^{2} W_{0}^{2} \widehat{\widetilde{K}}(0)^{2}+\beta \int_{B_{\Gamma}} d \mathbf{k} \frac{\widehat{\widetilde{K}}(\mathbf{k})^{2}}{\widehat{\widetilde{K}}(\mathbf{k})-\widehat{\widetilde{K}}(0)} .
\end{aligned}
$$

Recalling that $\widetilde{K}=\tilde{J}^{-1}$, this inequality is seen to imply that

$$
0 \leq(\mathbf{S}(0), \mathbf{S}(0))_{\beta, \mu} \leq \int_{B_{\Gamma}} \frac{d \mathbf{k}}{\tilde{\tilde{J}}(0)-\tilde{\widetilde{J}}(\mathbf{k})}+\beta M^{2},
$$

\footnotetext{
${ }^{7}$ This claim is heuristic, but can be replaced by an equivalent, mathematically rigorous one. ${ }^{(20)}$
} 
where $(\mathbf{S}(0), \mathbf{S}(0))_{\beta, \mu}$ is the Duhamel two-point function

$$
(\mathbf{S}(0), \mathbf{S}(0))_{\beta, \mu}=\int_{0}^{\beta}\langle\mathbf{S}(0,0) \cdot \mathbf{S}(0, t)\rangle_{\beta, \mu} d t,
$$

and

$$
M=W_{0} \widehat{\widetilde{K}}(0)
$$

is the spontaneous magnetization. Inequality (5.34) reproduces the infrared bounds in Ref. 4. For short-range exchange couplings, $0 \leq \hat{\widetilde{J}}(0)-\hat{\widetilde{J}}(\mathbf{k}) \leq$ const $|\mathbf{k}|^{2}$. Then, in dimension $d \geq 3$, and for $\mathcal{N} \geq 1$ Fermion species of spin $s \geq 1 / 2$, inequality (5.34) combined with an elementary lower bound on $(\mathbf{S}(0), \mathbf{S}(0))_{\beta, \mu}$, due to Bruch and Falk, shows that, for large $\beta(\mu=0)$,

$$
M>0 .
$$

See Ref. 4 for details.

Hence, by (3.42) and (5.29), and under the above assumptions on $J, d, \mathcal{N}$ and $s$,

$$
\begin{aligned}
\lim _{\varepsilon \rightarrow 0}\langle\mathbf{S}(x, 0)\rangle_{\beta, \mu, \varepsilon \mathbf{n}} & =-\sum_{y} K(x-y) \lim _{\varepsilon \rightarrow 0}\langle\mathbf{W}(y, 0)\rangle_{\beta, \mu, \varepsilon \mathbf{n}} \\
& =-(-1)^{|x|} \sum_{y} \widetilde{K}(x-y) \lim _{\varepsilon \rightarrow 0}\langle\mathbf{N}(y, 0)\rangle_{\beta, \mu, \varepsilon \mathbf{n}} \\
& =-(-1)^{|x|} M \mathbf{n} \neq 0,
\end{aligned}
$$

i.e., the system exhibits Néel order for sufficiently large values of $\beta$.

Arguments related to those used in the proof of the Mermin-Wagner theorem (see Ref. 6) show that if $M>0$ then there are gapless spin waves (see e.g. Ref. 31), in accordance with the mean-field picture of Section 4.

Next, we exploit Result F. It implies that, under condition (5.23), the following infrared bounds hold.

(IRF) Under the hypotheses of Result $\mathrm{F}$ (in particular, $J$ ferromagnetic and (5.23) holds), the inequalities

$$
\begin{aligned}
0 & \leq\left\langle\widehat{\mathbf{W}}\left(\mathbf{k}, k_{0}\right) \cdot \widehat{\mathbf{W}}\left(-\mathbf{k},-k_{0}\right)\right\rangle_{\beta, \mu} \\
& \leq W_{0}^{2} \delta_{0}(\mathbf{k}) \delta_{k_{0}, 0}+\beta^{-1}(\widehat{K}(\mathbf{k})-\widehat{K}(0))^{-1}
\end{aligned}
$$

for a constant $W_{0}^{2} \geq 0$, are valid. (See Refs. 5, 20, 21). The consequences of these inequalities are perfectly analogous to those discussed for antiferromagnets above. 
We set

$$
\mathbf{S}(x, t)=\sum_{a=1}^{\mathcal{M}}\left(\mathbf{S}^{(a)}(x, t)-\mathbf{S}^{(a+\mathcal{M})}(x, t)\right)
$$

see (5.23). Then, under appropriate conditions on $U_{0}, \mu$ and $K^{-1}(0)=J(0)$, and for $d \geq 3$,

$$
M=\lim _{\varepsilon \rightarrow 0}\langle\mathbf{S}(x, t)\rangle_{\beta, \mu, \varepsilon \mathbf{n}}=-\widehat{K}(0) W_{0} \mathbf{n} \neq 0
$$

for $\beta$ and $\mathcal{M}$ large enough, which proves long-range order at low temperatures, for $\mathcal{M}$ large enough; (presumably $\mathcal{N}=2 \mathcal{M}=2$ suffices if $J$ is nearest-neighbour and $d \geq 3$ ).

Unfortunately, there is, as yet, no such result on the existence of a phase transition in a quantum Heisenberg ferromagnet with only one species of fermions (electrons), i.e., $\mathcal{N}=1$. The reason is that this model is not described by a reflection-positive distribution, $d \mu_{\mathrm{eff}}(\mathbf{W})$, for the exchange field $\mathbf{W}$. Setting

$$
f_{x}(\mathbf{W})=\operatorname{det}\left(D_{\mathbf{W}(x, \cdot)}\right),
$$

$d \mu_{\mathrm{eff}}$ is given by

$$
d \mu_{\mathrm{eff}}(\mathbf{W})=\mathrm{const} \cdot \prod_{x} f_{x}(\mathbf{W}) d \mu_{K}(\mathbf{W})
$$

Now, if $J$ is ferromagnetic $d \mu_{K}(\mathbf{W})$ is reflection-positive if we set $\mathbf{W}_{\vartheta}(x, t)=$ $\mathbf{W}(\vartheta x, t)$. However, using (4.27),

$$
\begin{aligned}
\overline{\Theta f_{x}(\mathbf{W})} & =\overline{\operatorname{det}\left(D_{\mathbf{W}(\vartheta x, \cdot)}\right)} \\
& =\operatorname{det}\left(D_{-\mathbf{W}(\vartheta x, \cdot)}\right) \\
& =f_{\vartheta x}(-\mathbf{W}) \neq f_{\vartheta x}(\mathbf{W}) .
\end{aligned}
$$

Thus, $d \mu_{\mathrm{eff}}(\mathbf{W})$, as given in (5.41), is not reflection-positive, and there is therefore no reason why the Infrared Bounds (IRF) should hold. A very similar problem is encountered in the study of Bose-Einstein condensation in lattice models of non-relativistic, interacting bosons.

In order to get some preliminary insights into phase transitions for the Heisenberg ferromagnet, one may consider the following "static approximation": one replaces $f_{x}(\mathbf{W})$ by

$$
f_{x}^{(0)}(\mathbf{W})=\operatorname{det}\left(D_{\mathbf{W}^{(0)}(x)}\right)
$$

where

$$
\mathbf{W}^{(0)}(x)=\frac{1}{\beta} \int_{0}^{\beta} d t \mathbf{W}(x, t) \quad\left(=\frac{1}{\beta} \mathbf{W}\left(x, k_{0}=0\right)\right),
$$


which is time-independent. Then

$$
\overline{\Theta f_{x}^{(0)}(\mathbf{W})}=f_{\vartheta x}^{(0)}(-\mathbf{W})=f_{\vartheta x}^{(0)}(\mathbf{W}),
$$

as is easily checked, and $f_{x}^{(0)}(\mathbf{W})>0$. Replacing $d \mu_{\mathrm{eff}}(\mathbf{W})$ by

$$
d \mu_{\mathrm{eff}}^{(0)}(\mathbf{W})=\prod_{x} f_{x}^{(0)}(\mathbf{W}) d \mu_{K}(\mathbf{W})
$$

we conclude that $d \mu_{\mathrm{eff}}^{(0)}(\mathbf{W})$ is reflection-positive if $J$ is ferromagnetic, hence the infrared bounds (5.37) hold, and we conclude that, in the static approximation, for appropriate choices of $U_{0}, \mu$ and $J(0)$, a phase transition accompanied by continuous symmetry breaking occurs at sufficiently low temperatures.

If one replaces electrons with spin $\frac{1}{2}$ by fermions with spin $s$, or by $2 s$ identical species of spin- $\frac{1}{2}$ fermions (with $v_{a}=1$, for all $a=1, \ldots, 2 s$ ), and if one then takes $s \rightarrow \infty$ (rescaling the spin operators, $\left.\mathbf{S}(x, t) \mapsto \frac{1}{s} \mathbf{S}(x, t)\right)$ then the static approximation becomes exact. This is not surprising, because the limit $s \rightarrow \infty$ corresponds to the classical limit, as shown in Ref. 32, and the spin-s Heisenberg model approaches the classical Heisenberg model for which the existence of a phase transition has been established in Ref. 5. Our formalism, involving the exchange field $\mathbf{W}$, offers a neat and simple way of recovering some of the results in Ref. 32; (we leave this as an exercise to the reader).

\section{Remarks:}

1. We have outlined a proof of existence of a phase transition for a class of Heisenberg antiferromagnets; (the proof is mathematically rigorous; some missing details can be inferred from Refs. 4, 5. Of course, this is not a surprise. In Ref. 4, Dyson, Lieb and Simon have already proven a similar result also using reflection positivity (albeit in a different manner). Their method of proof, too, breaks down for quantum ferromagnets.

2. It appears that to understand phase transitions in quantum ferromagnets and the related phenomenon of Bose-Einstein condensation for lattice gases of non-relativistic bosons in a mathematically rigorous way, we would have to resort to a full-fledged renormalization group analysis-a technically rather demanding task.

3. The fact that the function $f_{x}(\mathbf{W})$ defined in (5.40) (see also (5.19)) is neither positive, nor even real is the origin of the "sign (complex phase) problem" in numerical simulations of quantum ferro- and antiferromagnets involving, e.g., the Monte Carlo method applied to (5.41). The great quality of methods based on reflection positivity is that they are applicable to the analysis of certain complex distributions $d \mu_{\mathrm{eff}}$. 
4. If one considers models in $d$ dimensions with $S O(2 n)$ spins, $n=$ $1,2,3, \ldots$, then for $d \geq 3$ one can prove a ferromagnetic phase transition, using arguments very similar to those described above.

\section{ACKNOWLEDGMENTS}

J. Fröhlich thanks S. Riesen and H.-C. Siegmann for very stimulating discussions of the experiments in Ref. 12, P. Wiegmann for some crucial advice with the calculations in Sec. 4, and the IHÉS for hospitality during much of the work on this paper. We all thank M. Azam for very useful and pleasant discussions on the uses of the exchange field.

\section{REFERENCES}

1. W. Pauli, Über Gasentartung und Paramagnetismus. Z. Physik 41:81 (1927).

2. P. W. Anderson, New approach to the theory of superexchange interactions. Phys. Rev. 115:2-13 (1959).

3. N. Datta, R. Fernández and J. Fröhlich, Effective Hamiltonians and phase diagrams for tightbinding models. J. Stat. Phys. 96:545-611 (1999).

4. F. Dyson, E. H. Lieb and B. Simon, Phase transitions in quantum spin systems with isotropic and non-isotropic interactions. J. Stat. Phys. 18:335 (1978).

5. J. Fröhlich, B. Simon and T. Spencer, Infrared Bounds, Phase Transitions And Continuous symmetry breaking. Commun. Math. Phys. 50:79 (1976).

6. N. D. Mermin, and H. Wagner, Absence of ferromagnetism or antiferromagnetism in one- or two-dimensional isotropic Heisenberg models. Phys. Rev. Lett. 17:1133-36 (1966).

7. W. Heisenberg. Z. Physik 49:619 (1928).

F. Bloch. Z. Physik 61:206 (1930).

E. C. Stoner. Proc. R. Soc. A165:372 (1938).

F. J. Dyson, General Theory of Spin-Wave Interactions. Phys. Rev. 102:1217-1230 (1956).

F. J. Dyson, Thermodynamic Behavior of an Ideal Ferromagnet. Phys. Rev. 102:1230-1244 (1956).

L. D. Landau, E. M. Lifshitz, Course of Theoretical Physics Volume 9, Statistical Physics, Part

2, by E. M. Lifshitz and L. P. Pitajewski, London: Pergamon 1981.

8. E. Brezin, J. C. Le Guillou and J. Zinn-Justin, Field Theoretical Approach To Critical Phenomena, in: Phase Transitions and Critical Phenomena, C. Domb and M. S. Green (Eds.) Volume 6, 125247, Academic PressLondon: 1976.

D. J. Amit, Field Theory, the Renormalization Group and Critical Phenomena, a.o.: McGraw-Hill New York, 1978.

9. A. Mielke, and H. Tasaki, Ferromagnetism in the Hubbard model. Commun. Math. Phys. 158:34171 (1993).

10. H. Tasaki, Ferromagnetism in the Hubbard model: A constructive approach. Commun. Math. Phys. 242:445-72 (2003).

11. J. Fröhlich, D. Ueltschi, Hund's Rule and Metallic Ferromagnetism. J. Stat. Phys. 118:973-996 (2005).

12. W. Weber, S. Riesen and H. C. Siegmann, Magnetization Precession by Hot Spin Injection, Science. 291:1015 (2001) and 
W. Weber, S. Riesen and H. C. Siegmann, Transmission of Electron Beams Through Thin Magnetic Films, in: Band-Ferromagnetism: Ground-State and Finite-Temperature Phenomena (Lecture Notes in Physics 580), K. Baberschke, M. Donath and W. Nolting (Eds.), Berlin, Heidelberg, Springer-Verlag New York: 2001.

13. D. Oberli, The ferromagnetic spin filter, PhD thesis ETH Zürich, Nr. 12933, (1998).

14. J. Fröhlich, U. M. Studer and E. Thiran, Quantum theory of large systems of nonrelativistic matter, in: Fluctuating geometries in statistical mechanics and field theory (Les Houches 1994), F. David, P. Ginsparg, and J. Zinn-Justin (Eds.), Elsevier 1996, [arXiv:cond-mat/9508062].

15. E. Fradkin, Field theories of condensed matter systems, Redwood City, a.o.: Addison-Wesley California, 1994.

16. J. Reuteler, QM particles scattering off a dynamical target, and decoherence, ETH-diploma thesis, spring 2005.

17. T. Chen, J. Fröhlich and M. Seifert. Renormalization group methods: Landau-Fermi liquid and BCS superconductor, in: Fluctuating geometries in statistical mechanics and field theory, F. David, P. Ginsparg, and J. Zinn-Justin (Eds.), Elsevier 1996, [arXiv:cond-mat/9508063].

18. M. Azam, L. Ferrari, J. Fröhlich, to appear.

19. H. Leutwyler, Nonrelativistic effective Lagrangians. Phys. Rev. D 49:3033 (1994), [arXiv:hep$\mathrm{ph} / 9311264]$

20. J. Fröhlich, R. Israel, E.H. Lieb, B. Simon, Phase Transitions and Reflection Positivity I. General Theory and Long Range Lattice Models. Commun. Math. Phys. 62:1-34, (1978).

21. J. Fröhlich, Mathematical Physics of Phase Transitions and Symmetry Breaking, Bulletin of the American Mathematical Society. 84:165-193 (1978).

22. M. N. Baibich, J. M. Broto, A. Fert, F. Nguyen Van Dau, F. Petroff P. Eitenne, G. Creuzet, A. Friederich and J. Chazelas, Giant Magnetoresistance of (001)Fe/(001)Cr Magnetic Superlattices. Phys. Rev. Lett. 61:2472 (1988).

P. C. van Son, H. van Kempen and P. Wyder, Boundary Resistance of the FerromagneticNonferromagnetic Metal Interface. Phys. Rev. Lett. 58:2271 (1987).

23. Y. Tserkovnyak, A. Brataas and G. E. W. Bauer, Enhanced Gilbert Damping in Thin Ferromagnetic Films. Phys. Rev. Lett. 88:117601 (2002).

24. L. Berger, Interaction of electrons with spin waves in the bulk and in multilayers. J. Appl. Phys. 91:6795 (2002).

25. I. N. Krivorotov, N. C. Emley, J. C. Sankey, S. I. Kiselev, D. C. Ralph and R. A. Buhrman, TimeDomain Measurements of Nanomagnet Dynamics Driven by Spin-Transfer Torques. Science 307:228 (2005).

26. W. H. Rippard and R. A. Buhrman, Spin-Dependent Hot Electron Transport in Co/Cu Thin Films. Phys. Rev. Lett. 84:971 (2000).

27. D. Ruelle, Statistical mechanics: rigorous results, Singapore: World Scientific, 1999.

28. L. Birke and J. Fröhlich, KMS, ETC. Rev. Math. Phys. 14:829-872 (2002), [arXiv: math-ph/ 0204023].

29. H. Chi and A. D. S. Nagi, Spin-wave velocity and specific heat of the Hubbard model at half filling with a path-integral approach, Phys. Rev. B46:8573 (1992).

30. V. Bach, E. H. Lieb, and M. V. Travaglia, Ferromagnetism of the Low-Density Hubbard Model in the Hartree-Fock Approximation, [arXiv:cond-mat/0506695].

D. Vollhardt, N. Blümer, K. Held, and M. Kollar, Metallic Ferromagnetism - An Electronic Correlation Phenomenon, in: Band-Ferromagnetism: Ground-State and Finite-Temperature Phenomena (Lecture Notes in Physics 580), K. Baberschke, M. Donath and W. Nolting (Eds.), Berlin, Heidelberg, Springer-Verlag New York: 2001.

31. J. Fröhlich and T. Spencer, Phase transitions in statistical mechanics and quantum field theory, in: New Developments in Quantum Field Theory and Statistical Mechanics; M. Lévy, and P. Mitter (Eds.), London: Plenum New York, 1977. 
32. E. H. Lieb, The Classical Limit of Quantum Spin Systems. Commun. Math. Phys. 31:327-340 (1973).

33. J. Derezinski, V. Jaksic and C. Pillet, Perturbation theory of $\mathrm{W}^{*}$-dynamics, Liouvilleans and KMS-states. Rev. Math. Phys. 15:447 (2003).

34. V. Bach, J. Fröhlich and I. M. Sigal. Return to equilibrium. J. Math. Phys. 41:3985 (2000) .

35. J. Fröhlich and M. Merkli, Another Return of 'Return to Equilibrium' Commun. Math. Phys. 251:235-262 (2004). 